\title{
Existence of global weak solutions to the compressible Ericksen-Leslie system in dimension one
}

\author{
Huajun Gong* Tao Huang ${ }^{\dagger}$ Changyou Wang ${ }^{\ddagger}$
}

\begin{abstract}
We consider the compressible Ericksen-Leslie system of liquid crystal flows in one dimension. A global weak solution is constructed with initial density $\rho_{0} \geq 0$ and $\rho_{0} \in L^{\gamma}$ for $\gamma>1$.
\end{abstract}

\section{Introduction}

Nematic liquid crystals are composed of rod-like molecules characterized by average alignment of the long axes of neighboring molecules, which have simplest structures among various types of liquid crystals. The dynamic theory of nematic liquid crystals has been first proposed by Ericksen [5] and Leslie [14] in the 1960's, which is a macroscopic continuum description of the time evolution of both flow velocity field and orientation order parameter of rod-like liquid crystals.

In this paper, we will study the compressible Ericksen-Leslie system of liquid crystal flows (see [19], [1] for modeling). Let $\Omega \subset \mathbb{R}^{3}$ be a bounded domain with smooth boundary, and $\mathbb{S}^{2}$ be the unit sphere in $\mathbb{R}^{3}$. The compressible Ericksen-Leslie system is given as follows

$$
\left\{\begin{array}{l}
\rho_{t}+\nabla \cdot(\rho \mathbf{u})=0 \\
\rho \dot{\mathbf{u}}+\nabla P=\nabla \cdot \sigma-\nabla \cdot\left(\frac{\partial W}{\partial \nabla \mathbf{n}} \otimes \nabla \mathbf{n}\right), \\
\mathbf{g}+\frac{\partial W}{\partial \mathbf{n}}-\nabla \cdot\left(\frac{\partial W}{\partial \nabla \mathbf{n}}\right)=\lambda \mathbf{n} .
\end{array}\right.
$$

Here, $\rho(\mathbf{x}, t): \Omega \times(0, \infty) \rightarrow \mathbb{R}$ is the density, $\mathbf{u}(\mathbf{x}, t): \Omega \times(0, \infty) \rightarrow \mathbb{R}^{3}$ is the fluid velocity field, $\mathbf{n}(\mathbf{x}, t): \Omega \times(0, \infty) \rightarrow \mathbb{S}^{2}$ is the orientation order parameters of nematic material. $\lambda$ is the Lagrangian multiplier of the constraint $|\mathbf{n}|=1, \dot{f}=f_{t}+\mathbf{u} \cdot \nabla f$ is the material derivative of function $f$, and $\mathbf{a} \otimes \mathbf{b}=\mathbf{a} \mathbf{b}^{T}$ for column vectors $\mathbf{a}$ and $\mathbf{b}$ in $\mathbb{R}^{3}$.

The macrostructure of the crystals has been determined by the Oseen-Frank energy density (cf. [20, 8]). One may take the Oseen-Frank energy density in the compressible case as

$$
\begin{aligned}
2 W(\rho, \mathbf{n}, \nabla \mathbf{n})= & \frac{2}{\gamma-1} \rho^{\gamma}+K_{1}(\operatorname{div} \mathbf{n})^{2}+K_{2}(\mathbf{n} \cdot \operatorname{curl} \mathbf{n})^{2}+K_{3}|\mathbf{n} \times \operatorname{curl} \mathbf{n}|^{2} \\
& +\left(K_{2}+K_{4}\right)\left[\operatorname{tr}(\nabla \mathbf{n})^{2}-(\operatorname{div} \mathbf{n})^{2}\right],
\end{aligned}
$$

*College of Mathematics and Statistics, Shenzhen University, Shenzhen 518060, Guangdong, China.

${ }^{\dagger}$ Department of Mathematics, Wayne State University, Detroit, MI 48202, USA.

${ }^{\ddagger}$ Department of Mathematics, Purdue University, West Lafayette, IN 47907, USA. 
where $\gamma>1$, and $K_{j}, j=1,2,3$, are the positive constants representing splay, twist, and bend effects respectively, with $K_{2} \geq\left|K_{4}\right|, 2 K_{1} \geq K_{2}+K_{4}$. Then the pressure can be given by the Maxwell relation

$$
P(\rho)=\rho W_{\rho}(\rho, \mathbf{n}, \nabla \mathbf{n})-W(\rho, \mathbf{n}, \nabla \mathbf{n}) .
$$

For simplicity, we only consider the case $K_{1}=K_{2}=K_{3}=1, K_{4}=0$ in this paper. The OseenFrank energy in the compressible case becomes

$$
2 W(\rho, \mathbf{n}, \nabla \mathbf{n})=\frac{2}{\gamma-1} \rho^{\gamma}+|\nabla \mathbf{n}|^{2} .
$$

Therefore

$$
\nabla \cdot\left(\frac{\partial W}{\partial \nabla \mathbf{n}} \otimes \nabla \mathbf{n}\right)=\nabla \cdot(\nabla \mathbf{n} \odot \nabla \mathbf{n}), \quad \frac{\partial W}{\partial \mathbf{n}}=0, \quad \nabla \cdot\left(\frac{\partial W}{\partial \nabla \mathbf{n}}\right)=\Delta \mathbf{n}, \quad P=\rho^{\gamma}-\frac{1}{2}|\nabla n|^{2} .
$$

Let

$$
D=\frac{1}{2}\left(\nabla \mathbf{u}+\nabla^{T} \mathbf{u}\right), \quad \omega=\frac{1}{2}\left(\nabla \mathbf{u}-\nabla^{T} \mathbf{u}\right)=\frac{1}{2}\left(\frac{\partial u^{i}}{\partial x_{j}}-\frac{\partial u^{j}}{\partial x_{i}}\right), \quad N=\dot{\mathbf{n}}-\omega \mathbf{n},
$$

represent the rate of strain tensor, skew-symmetric part of the strain rate, and the rigid rotation part of director changing rate by fluid vorticity, respectively. The kinematic transport $\mathbf{g}$ is given by

$$
\mathbf{g}=\gamma_{1} N+\gamma_{2} D \mathbf{n}-\gamma_{2}\left(\mathbf{n}^{T} D \mathbf{n}\right) \mathbf{n}
$$

which represents the effect of the macroscopic flow field on the microscopic structure. The material coefficients $\gamma_{1}$ and $\gamma_{2}$ reflect the molecular shape and the slippery part between fluid and particles. The first term of $\mathbf{g}$ represents the rigid rotation of molecules, while the second term stands for the stretching of molecules by the flow. The viscous (Leslie) stress tensor $\sigma$ has the following form (cf. [15] [1])

$$
\begin{aligned}
\sigma= & \alpha_{0}\left(\mathbf{n}^{T} D \mathbf{n}\right) \mathbb{I}+\alpha_{1}\left(\mathbf{n}^{T} D \mathbf{n}\right) \mathbf{n} \otimes \mathbf{n}+\alpha_{2} N \otimes \mathbf{n}+\alpha_{3} \mathbf{n} \otimes N \\
& +\alpha_{4} D+\alpha_{5}(D \mathbf{n}) \otimes \mathbf{n}+\alpha_{6} \mathbf{n} \otimes(D \mathbf{n})+\alpha_{7}(\operatorname{tr} D) \mathbb{I}+\alpha_{8}(\operatorname{tr} D) \mathbf{n} \otimes \mathbf{n} .
\end{aligned}
$$

These coefficients $\alpha_{j}(0 \leq j \leq 8)$, depending on material and temperature, are called Leslie coefficients. The following relations are often assumed in the literature.

$$
\gamma_{1}=\alpha_{3}-\alpha_{2}, \quad \gamma_{2}=\alpha_{6}-\alpha_{5}, \quad \alpha_{2}+\alpha_{3}=\alpha_{6}-\alpha_{5}
$$

The first two relations are compatibility conditions, while the third relation is called Parodi's relation, derived from Onsager reciprocal relations expressing the equality of certain relations between flows and forces in thermodynamic systems out of equilibrium (cf. [21]). They also satisfy the following empirical relations (cf. [15], [1])

$$
\begin{aligned}
& \alpha_{4}>0, \quad 2 \alpha_{1}+3 \alpha_{4}+2 \alpha_{5}+2 \alpha_{6}>0, \quad \gamma_{1}=\alpha_{3}-\alpha_{2}>0, \\
& 2 \alpha_{4}+\alpha_{5}+\alpha_{6}>0, \quad 4 \gamma_{1}\left(2 \alpha_{4}+\alpha_{5}+\alpha_{6}\right)>\left(\alpha_{2}+\alpha_{3}+\gamma_{2}\right)^{2} \\
& \alpha_{4}+\alpha_{7}>\alpha_{1}+\frac{\gamma_{2}^{2}}{\gamma_{1}} \geq 0 \\
& 2 \alpha_{4}+\alpha_{5}+\alpha_{6}-\frac{\gamma_{2}^{2}}{\gamma_{1}}>\alpha_{0}+\alpha_{1}+\alpha_{5}+\alpha_{6}+\alpha_{8} \geq 0 .
\end{aligned}
$$


It is easy to see that an example of coefficients $\alpha_{1}, \cdots, \alpha_{8}$ satisfying (1.5) and (1.6) can be taken as follows

$$
\alpha_{0}=\alpha_{1}=\alpha_{5}=\alpha_{6}=\alpha_{7}=\alpha_{8}=0, \quad \alpha_{2}=-1, \quad \alpha_{3}=\alpha_{4}=1
$$

so that

$$
\gamma_{1}=\alpha_{3}-\alpha_{2}=2>0, \quad \gamma_{2}=\alpha_{6}-\alpha_{5}=\alpha_{2}+\alpha_{3}=0
$$

A simplified compressible Ericksen-Leslie system has been recently studied. The idea of simplification was first proposed for the incompressible system by Lin in [16]. In dimension one, the global strong and weak solutions have been constructed in [3] and [4]. In dimension two, under the assumption that the initial data of $\mathbf{n}$ is contained in $\mathbb{S}_{+}^{2}$, global weak solutions have been constructed in [11]. In dimension three, the local existence of strong solutions has been studied by [9] and [10], and when the initial data of $\mathbf{n}$ is contained in $\mathbb{S}_{+}^{2}$, global weak solutions have been constructed in [17. The incompressible limit of compressible nematic liquid crystal flows has been studied by [2].

We also mention a related work [12], in which the EricksenLeslies parabolichyperbolic liquid crystal model has been studied. For small initial data, they have shown the existence of global solutions in dimension three.

\subsection{One dimensional model and statement of main results}

One of the main motivations of this paper is to investigate the impact of general Leslie stress tensors to the solutions of the compressible Ericksen-Leslie system with coefficients satisfying algebraic conditions (1.5) and (1.6) ensuring the energy dissipation property. Because of the technical complexity of the Ericksen-Leslie system in higher dimensions, we will only consider the following simpler case in one dimension, in which the director field $\mathbf{n}$ is assumed to map into the equator $\mathbb{S}^{1}$,

$$
\mathbf{u}=(u(x, t), v(x, t), 0)^{T}, \quad \mathbf{n}=(\cos n(x, t), \sin n(x, t), 0)^{T}
$$

for any $x \in[0,1]$ and $t \in(0, \infty)$. From the derivation given by Section 2 below, the system (1.1) becomes

$$
\left\{\begin{array}{l}
\rho_{t}+(\rho u)_{x}=0 \\
(\rho u)_{t}+\left(\rho u^{2}\right)_{x}+\left(\rho^{\gamma}\right)_{x}=J^{1}-n_{x x} n_{x} \\
(\rho v)_{t}+(\rho u v)_{x}=J^{2} \\
\gamma_{1}\left(\dot{n}-\frac{1}{2} v_{x}\right)-\gamma_{2}\left(u_{x} \cos n \sin n+\frac{1}{2} v_{x}\left(1-2 \cos ^{2} n\right)\right)=n_{x x}
\end{array}\right.
$$

Here

$$
\begin{aligned}
J^{1}= & \left(\alpha_{0}+\alpha_{5}+\alpha_{6}+\alpha_{8}\right)\left(u_{x} \cos ^{2} n\right)_{x}+\alpha_{1}\left(u_{x} \cos ^{4} n\right)_{x}-\left(\alpha_{2}+\alpha_{3}\right)(\dot{n} \cos n \sin n)_{x}+\left(\alpha_{4}+\alpha_{7}\right) u_{x x} \\
& +\alpha_{0}\left(v_{x} \cos n \sin n\right)_{x}+\alpha_{1}\left(v_{x} \cos ^{3} n \sin n\right)_{x}+\frac{1}{2}\left(\alpha_{2}+\alpha_{3}+\alpha_{5}+\alpha_{6}\right)\left(v_{x} \cos n \sin n\right)_{x},
\end{aligned}
$$

and

$$
\begin{aligned}
J^{2}= & \alpha_{1}\left(u_{x} \cos ^{3} n \sin n\right)_{x}+\alpha_{2}\left(\dot{n} \cos ^{2} n\right)_{x}-\alpha_{3}\left(\dot{n} \sin ^{2} n\right)_{x}+\left(\alpha_{6}+\alpha_{8}\right)\left(u_{x} \cos n \sin n\right)_{x} \\
& +\alpha_{1}\left(v_{x} \cos ^{2} n \sin ^{2} n\right)_{x}+\frac{1}{2}\left(-\alpha_{2}+\alpha_{5}\right)\left(v_{x} \cos ^{2} n\right)_{x}+\frac{1}{2}\left(\alpha_{3}+\alpha_{6}\right)\left(v_{x} \sin ^{2} n\right)_{x}+\frac{1}{2} \alpha_{4} v_{x x} .
\end{aligned}
$$


For this system, we consider the following initial and boundary values

$$
\begin{gathered}
(\rho, \rho u, \rho v, n)(x, 0)=\left(\rho_{0}, m_{0}, l_{0}, n_{0}\right)(x), \\
u(0, t)=v(0, t)=u(1, t)=v(1, t)=0, \quad n_{x}(0, t)=n_{x}(1, t)=0 .
\end{gathered}
$$

Denote the energy of the system (1.7) by

$$
\mathcal{E}(t):=\frac{1}{2} \int_{0}^{1} \rho\left(u^{2}+v^{2}\right)+\frac{1}{\gamma-1} \int_{0}^{1} \rho^{\gamma}+\frac{1}{2} \int_{0}^{1} n_{x}^{2}
$$

For any smooth solution $(\rho, u, v, n)$, the energy functional satisfies the following energy inequality, whose proof will be provided in Section 3,

$$
\begin{aligned}
\frac{d}{d t} \mathcal{E}(t) & =-\mathcal{D} \\
& :=-\int_{0}^{1}\left[\sqrt{\gamma_{1}} \dot{n}-\frac{1}{2}\left(\frac{\gamma_{2}}{\sqrt{\gamma_{1}}} u_{x} \sin (2 n)+\frac{1}{\sqrt{\gamma_{1}}}\left(\gamma_{1}-\gamma_{2} \cos (2 n)\right) v_{x}\right)\right]^{2} \\
& -\int_{0}^{1}\left[\frac{1}{4}\left(-\alpha_{1}-\frac{\gamma_{2}^{2}}{\gamma_{1}}\right) u_{x}^{2}+\left(\alpha_{4}+\alpha_{7}\right) u_{x}^{2}\right]-\frac{1}{4} \int_{0}^{1}\left(2 \alpha_{4}+\alpha_{5}+\alpha_{6}-\frac{\gamma_{2}^{2}}{\gamma_{1}}\right) v_{x}^{2} \\
& -\frac{1}{4}\left(\alpha_{1}+\frac{\gamma_{2}^{2}}{\gamma_{1}}\right) \int_{0}^{1}\left(u_{x} \cos (2 n)+v_{x} \sin (2 n)\right)^{2} \\
& -\left(\alpha_{0}+\alpha_{1}+\alpha_{5}+\alpha_{6}+\alpha_{8}\right) \int_{0}^{1}\left[\left(u_{x} \cos n+\frac{1}{2} v_{x} \sin n\right)^{2}-\frac{1}{4} v_{x}^{2} \sin ^{2} n\right] .
\end{aligned}
$$

By the assumptions (1.6) on coefficients, the system (1.7) is dissipative.

Definition 1.1 For any time $0<T<\infty$, a collection of functions $(\rho, u, v, n)(x, t)$ is a global weak solution to the initial and boundary value problem (1.7)-(1.9) if

$$
\begin{gathered}
\rho \geq 0, \text { a.e., } \quad \rho \in L^{\infty}\left(0, T ; L^{\gamma}\right), \quad \rho u^{2}, \rho v^{2} \in L^{\infty}\left(0, T ; L^{1}\right), \quad u, v \in L^{2}\left(0, T ; H_{0}^{1}\right) \\
n \in L^{\infty}\left(0, T ; H^{1}\right) \cap L^{2}\left(0, T ; H^{2}\right), \quad n_{t} \in L^{2}\left(0, T ; L^{2}\right) .
\end{gathered}
$$

(2) The equations of $\rho, u, v$ are satisfied in the weak sense, while the equation of $n$ is valid a.e.. The initial condition (1.8) is satisfied in the weak sense.

(3) The energy inequality is valid for a.e. $t \in(0, T)$

$$
\mathcal{E}(t)+\int_{0}^{t} \mathcal{D} \leq \mathcal{E}_{0}=\frac{1}{2} \int_{0}^{1} \frac{m_{0}^{2}+l_{0}^{2}}{\rho_{0}}+\frac{1}{\gamma-1} \int_{0}^{1} \rho_{0}^{\gamma}+\frac{1}{2} \int_{0}^{1}\left(n_{0}\right)_{x}^{2} .
$$

The following is the main results in this paper.

Theorem 1.2 Assume that the coefficients of Leslie stress tensor satisfy the algebraic conditions (1.5) and (1.6). Then, for any $0<T<\infty$ and any initial data

$$
0 \leq \rho_{0} \in L^{\gamma}, \quad \frac{m_{0}}{\sqrt{\rho_{0}}}, \quad \frac{l_{0}}{\sqrt{\rho_{0}}} \in L^{2}, \quad n_{0} \in H^{1},
$$

there is a global weak solution $(\rho, u, v, n)(x, t)$ on $(0,1) \times(0, T)$ to the initial and boundary value problem (1.7)-(1.9). Furthermore, $\rho \in L^{2 \gamma}((0,1) \times(0, T))$. 
The main ideas of the proof utilize and extend those from [7], [13], and [6] in the study of the compressible Navier-Stokes equations, where the quantity called effective viscous flux has played crucial roles in controlling the oscillation of the density function $\rho$. However, the general Leslie stress tensors in the compressible Ericksen-Leslie system (1.7) induce two complicate second-order terms $J^{1}$ and $J^{2}$ that prohibit direct applications of the method of effective viscous flux. In this paper, we observe that with the algebraic conditions (1.5) and (1.6), the system of $\mathbf{u}=(u, v)^{T}$ can still be shown to be uniformly parabolic (see (5.25) and (5.26) below), i.e. the coefficient matrix of the second-order terms is uniformly elliptic. Using the inverse of coefficient matrix of the secondorder terms, we can then define a modified form of effective viscous flux as in Lemma 5.3, which yields the desired estimates that are necessary in the limiting process of approximated solutions.

The paper is organized as follows. In Section 2, we will sketch a derivation of the system (1.7). In Section 3, we will derive some a priori estimates for smooth solutions of (1.7). In Section 4, an approximated system will be introduced, and the existence of global regular solutions of this approximated system will be proven. In Section 5, we will prove the existence of global weak solutions through some delicate analysis of the convergence process.

\section{Derivation of the model in one dimension}

This section is devoted to the derivation of the system (1.7) in dimension one. If a solution takes the form

$$
\mathbf{u}=(u(x, t), v(x, t))^{T}, \quad \mathbf{n}=(\cos n(x, t), \sin n(x, t))^{T},(x, t) \in(0,1) \times(0, T),
$$

then

$$
\nabla \mathbf{u}=\left[\begin{array}{cc}
u_{x} & 0 \\
v_{x} & 0
\end{array}\right], \quad \nabla^{T} \mathbf{u}=\left[\begin{array}{cc}
u_{x} & v_{x} \\
0 & 0
\end{array}\right]
$$

so that

$$
\begin{gathered}
D=\left[\begin{array}{cc}
u_{x} & \frac{1}{2} v_{x} \\
\frac{1}{2} v_{x} & 0
\end{array}\right] \quad \omega=\left[\begin{array}{cc}
0 & -\frac{1}{2} v_{x} \\
\frac{1}{2} v_{x} & 0
\end{array}\right], \\
\operatorname{tr} D=u_{x}, \quad N=\dot{\mathbf{n}}-\omega \mathbf{n}=\left(\dot{n}-\frac{1}{2} v_{x}\right)(-\sin n, \cos n)^{T} .
\end{gathered}
$$

Direct calculations imply that

$$
\begin{gathered}
D \mathbf{n}=\left(u_{x} \cos n+\frac{1}{2} v_{x} \sin n, \frac{1}{2} v_{x} \cos n\right)^{T}, \quad \mathbf{n}^{T} D \mathbf{n}=u_{x} \cos ^{2} n+v_{x} \cos n \sin n, \\
\mathbf{n} \otimes \mathbf{n}=\left[\begin{array}{cc}
\cos ^{2} n & \cos n \sin n \\
\cos n \sin n & \sin ^{2} n
\end{array}\right], \\
\left(\mathbf{n}^{T} D \mathbf{n}\right) \mathbf{n} \otimes \mathbf{n}=\left(u_{x} \cos ^{2} n+v_{x} \cos n \sin n\right)\left[\begin{array}{cc}
\cos ^{2} n & \cos n \sin n \\
\cos n \sin n & \sin ^{2} n
\end{array}\right],
\end{gathered}
$$




$$
\begin{gathered}
N \otimes \mathbf{n}=\left(\dot{n}-\frac{1}{2} v_{x}\right)\left[\begin{array}{cc}
-\cos n \sin n & -\sin ^{2} n \\
\cos ^{2} n & \cos n \sin n
\end{array}\right], \\
\mathbf{n} \otimes N=\left(\dot{n}-\frac{1}{2} v_{x}\right)\left[\begin{array}{cc}
-\cos n \sin n & \cos ^{2} n \\
-\sin ^{2} n & \cos n \sin n
\end{array}\right], \\
(D \mathbf{n}) \otimes \mathbf{n}=\left[\begin{array}{cc}
u_{x} \cos ^{2} n+\frac{1}{2} v_{x} \cos n \sin n & u_{x} \cos n \sin n+\frac{1}{2} v_{x} \sin ^{2} n \\
\frac{1}{2} v_{x} \cos ^{2} n & \frac{1}{2} v_{x} \cos n \sin n
\end{array}\right], \\
\mathbf{n} \otimes(D \mathbf{n})=\left[\begin{array}{cc}
u_{x} \cos ^{2} n+\frac{1}{2} v_{x} \cos n \sin n & \frac{1}{2} v_{x} \cos ^{2} n \\
u_{x} \cos n \sin n+\frac{1}{2} v_{x} \sin { }^{2} n & \frac{1}{2} v_{x} \cos n \sin n
\end{array}\right] .
\end{gathered}
$$

Hence

$$
\nabla \cdot \sigma=\left(J^{1}, J^{2}\right)^{T}
$$

where

$$
\begin{aligned}
J^{1}= & \left(\alpha_{0}+\alpha_{5}+\alpha_{6}+\alpha_{8}\right)\left(u_{x} \cos ^{2} n\right)_{x}+\alpha_{1}\left(u_{x} \cos ^{4} n\right)_{x}-\left(\alpha_{2}+\alpha_{3}\right)(\dot{n} \cos n \sin n)_{x}+\left(\alpha_{4}+\alpha_{7}\right) u_{x x} \\
& +\alpha_{0}\left(v_{x} \cos n \sin n\right)_{x}+\alpha_{1}\left(v_{x} \cos ^{3} n \sin n\right)_{x}+\frac{1}{2}\left(\alpha_{2}+\alpha_{3}+\alpha_{5}+\alpha_{6}\right)\left(v_{x} \cos n \sin n\right)_{x},
\end{aligned}
$$

and

$$
\begin{aligned}
J^{2}= & \alpha_{1}\left(u_{x} \cos ^{3} n \sin n\right)_{x}+\alpha_{2}\left(\dot{n} \cos ^{2} n\right)_{x}-\alpha_{3}\left(\dot{n} \sin ^{2} n\right)_{x}+\left(\alpha_{6}+\alpha_{8}\right)\left(u_{x} \cos n \sin n\right)_{x} \\
& +\alpha_{1}\left(v_{x} \cos ^{2} n \sin ^{2} n\right)_{x}+\frac{1}{2}\left(-\alpha_{2}+\alpha_{5}\right)\left(v_{x} \cos ^{2} n\right)_{x}+\frac{1}{2}\left(\alpha_{3}+\alpha_{6}\right)\left(v_{x} \sin ^{2} n\right)_{x}+\frac{1}{2} \alpha_{4} v_{x x} .
\end{aligned}
$$

The terms related to $\mathbf{n}$ can be computed as follows

$$
\begin{gathered}
\mathbf{n}_{t}=n_{t}(-\sin n, \cos n)^{T}, \\
\mathbf{n}_{x}=n_{x}(-\sin n, \cos n)^{T}, \quad\left|\mathbf{n}_{x}\right|^{2}=\left(n_{x}\right)^{2} \\
\mathbf{u} \cdot \mathbf{n}=u \mathbf{n}_{x}=u n_{x}(-\sin n, \cos n)^{T} \\
\mathbf{n}_{x x}=n_{x x}(-\sin n, \cos n)^{T}+\left(n_{x}\right)^{2}(-\cos n,-\sin n)^{T}, \\
\nabla \cdot(\nabla \mathbf{n} \odot \nabla \mathbf{n})-\frac{1}{2} \nabla|\nabla \mathbf{n}|^{2}=\Delta \mathbf{n} \nabla \mathbf{n}=\left(n_{x x} n_{x}, 0\right)^{T} .
\end{gathered}
$$

Therefore, $u(x, t)$ satisfies

$$
\rho u_{t}+\rho u u_{x}+\left(\rho^{\gamma}\right)_{x}=J^{1}-n_{x x} n_{x},
$$

and $v(x, t)$ satisfies

$$
\rho v_{t}+\rho u v_{x}=J^{2} .
$$


Now we can calculate the equation of $n$ as follows.

$$
\begin{aligned}
\mathbf{g}= & \gamma_{1} N+\gamma_{2} D \mathbf{n}-\gamma_{2}\left(\mathbf{n}^{T} D \mathbf{n}\right) \mathbf{n} \\
& =\gamma_{1}\left(\dot{n}-\frac{1}{2} v_{x}\right)(-\sin n, \cos n)^{T}+\gamma_{2}\left(u_{x} \cos n+\frac{1}{2} v_{x} \sin n, \frac{1}{2} v_{x} \cos n\right)^{T} \\
& -\gamma_{2}\left(u_{x} \cos ^{2} n+v_{x} \cos n \sin n\right)(\cos n, \sin n)^{T} \\
& =\gamma_{1}\left(\dot{n}-\frac{1}{2} v_{x}\right)(-\sin n, \cos n)^{T} \\
& +\gamma_{2}\left(u_{x} \cos n \sin ^{2} n+\frac{1}{2} v_{x} \sin n\left(1-2 \cos ^{2} n\right),-u_{x} \cos ^{2} n \sin n+\frac{1}{2} v_{x} \cos n\left(1-2 \sin ^{2} n\right)\right)^{T} \\
& =\gamma_{1}\left(\dot{n}-\frac{1}{2} v_{x}\right)(-\sin n, \cos n)^{T} \\
& -\gamma_{2}\left(u_{x} \cos n \sin n+\frac{1}{2} v_{x}\left(1-2 \cos ^{2} n\right)\right)(-\sin n, \cos n)^{T}, \\
& \lambda \mathbf{n}=\left(|\nabla \mathbf{n}|^{2}+\gamma_{1} N \cdot \mathbf{n}\right) \mathbf{n}=\left(n_{x}\right)^{2}(\cos n, \sin n)^{T} .
\end{aligned}
$$

Therefore $n(x, t)$ satisfies

$$
\gamma_{1}\left(\dot{n}-\frac{1}{2} v_{x}\right)-\gamma_{2}\left(u_{x} \cos n \sin n+\frac{1}{2} v_{x}\left(1-2 \cos ^{2} n\right)\right)=n_{x x} .
$$

Thus the system (1.1) reduces to (1.7).

\section{A priori estimates}

In this section, we will prove several useful a priori estimates for smooth solutions of system (1.7).

Lemma 3.1 Any smooth solution to the system (1.7) satisfies the following energy inequality

$$
\begin{aligned}
\frac{d}{d t} \mathcal{E}(t)= & -\int_{0}^{1}\left[\sqrt{\gamma_{1}} \dot{n}-\frac{1}{2}\left(\frac{\gamma_{2}}{\sqrt{\gamma_{1}}} u_{x} \sin (2 n)+\frac{1}{\sqrt{\gamma_{1}}}\left(\gamma_{1}-\gamma_{2} \cos (2 n)\right) v_{x}\right)\right]^{2} \\
& -\int_{0}^{1}\left[\frac{1}{4}\left(-\alpha_{1}-\frac{\gamma_{2}^{2}}{\gamma_{1}}\right) u_{x}^{2}+\left(\alpha_{4}+\alpha_{7}\right) u_{x}^{2}\right]-\frac{1}{4} \int_{0}^{1}\left(2 \alpha_{4}+\alpha_{5}+\alpha_{6}-\frac{\gamma_{2}^{2}}{\gamma_{1}}\right) v_{x}^{2} \\
& -\frac{1}{4}\left(\alpha_{1}+\frac{\gamma_{2}^{2}}{\gamma_{1}}\right) \int_{0}^{1}\left(u_{x} \cos (2 n)+v_{x} \sin (2 n)\right)^{2} \\
& -\left(\alpha_{0}+\alpha_{1}+\alpha_{5}+\alpha_{6}+\alpha_{8}\right) \int_{0}^{1}\left[\left(u_{x} \cos n+\frac{1}{2} v_{x} \sin n\right)^{2}-\frac{1}{4} v_{x}^{2} \sin ^{2} n\right] .
\end{aligned}
$$

Proof. Multiplying the second equation by $u$, the third equation by $v$ and integrating over $[0,1]$, we have

$$
\frac{1}{2} \frac{d}{d t} \int_{0}^{1} \rho\left(u^{2}+v^{2}\right)+\frac{1}{\gamma-1} \frac{d}{d t} \int_{0}^{1} \rho^{\gamma}=\int_{0}^{1}\left(J^{1} u+J^{2} v-u n_{x x} n_{x}\right)
$$


Multiplying the last equation by $\dot{n}$ and integrating over $[0,1]$, we obtain

$$
\frac{d}{d t} \frac{1}{2} \int_{0}^{1}\left(n_{x}\right)^{2}+\gamma_{1} \int_{0}^{1} \dot{n}^{2}=\int_{0}^{1}\left[\frac{1}{2} \gamma_{2} u_{x} \sin (2 n) \dot{n}+\frac{1}{2}\left(\gamma_{1}-\gamma_{2} \cos (2 n)\right) v_{x} \dot{n}+u n_{x x} n_{x}\right] .
$$

Adding these two equations together, we have

$$
\begin{aligned}
& \frac{1}{2} \frac{d}{d t} \int_{0}^{1} \rho\left(u^{2}+v^{2}\right)+\frac{1}{\gamma-1} \frac{d}{d t} \int_{0}^{1} \rho^{\gamma}+\frac{1}{2} \frac{d}{d t} \int_{0}^{1}\left(n_{x}\right)^{2} \\
= & \int_{0}^{1}\left(J^{1} u+J^{2} v\right)-\gamma_{1} \int_{0}^{1} \dot{n}^{2}+\int_{0}^{1} \frac{1}{2}\left[\gamma_{2} u_{x} \sin (2 n) \dot{n}+\left(\gamma_{1}-\gamma_{2} \cos (2 n)\right) v_{x} \dot{n}\right] .
\end{aligned}
$$

By integrating by parts, we can estimate the term related to $J^{1}, J^{2}$ as follows

$$
\begin{aligned}
& \int_{0}^{1} J^{1} u \\
= & -\int_{0}^{1}\left[\left(\alpha_{0}+\alpha_{5}+\alpha_{6}+\alpha_{8}\right) u_{x}^{2} \cos ^{2} n+\alpha_{1} u_{x}^{2} \cos ^{4} n+\left(\alpha_{4}+\alpha_{7}\right) u_{x}^{2}\right] \\
& -\int_{0}^{1}\left[\alpha_{0} u_{x} v_{x} \cos n \sin n+\alpha_{1} u_{x} v_{x} \cos ^{3} n \sin n+\frac{1}{2}\left(\alpha_{2}+\alpha_{3}+\alpha_{5}+\alpha_{6}\right) u_{x} v_{x} \cos n \sin n\right] \\
+ & \int_{0}^{1}\left(\alpha_{2}+\alpha_{3}\right) u_{x} \dot{n} \cos n \sin n, \\
& \int_{0}^{1} J^{2} v \\
= & -\int_{0}^{1}\left[\alpha_{1} v_{x}^{2} \cos ^{2} n \sin ^{2} n+\frac{1}{2}\left(-\alpha_{2}+\alpha_{5}\right) v_{x}^{2} \cos ^{2} n+\frac{1}{2}\left(\alpha_{3}+\alpha_{6}\right) v_{x}^{2} \sin ^{2} n+\frac{1}{2} \alpha_{4} v_{x}^{2}\right] \\
& -\int_{0}^{1}\left[\alpha_{1} u_{x} v_{x} \cos ^{3} n \sin n+\left(\alpha_{6}+\alpha_{8}\right) u_{x} v_{x} \cos _{n} \sin n\right] \\
& -\int_{0}^{1}\left[\alpha_{2} v_{x} \dot{n} \cos ^{2} n-\alpha_{3} v_{x} \dot{n} \sin ^{2} n\right] .
\end{aligned}
$$

First notice that all the terms related to $\alpha_{1}$ in (3.3) and (3.4) can be written as

$$
\begin{array}{r}
-\alpha_{1} \int_{0}^{1}\left[u_{x}^{2} \cos ^{4} n+2 u_{x} v_{x} \cos ^{3} n \sin n+v_{x}^{2} \cos ^{2} n \sin ^{2} n\right] \\
=-\alpha_{1} \int_{0}^{1}\left[u_{x} \cos ^{2} n+v_{x} \cos n \sin n\right]^{2} .
\end{array}
$$

The other term related to $u_{x} v_{x}$ in (3.3) and (3.4) (without terms with $\alpha_{1}$ ) can be written as

$$
\begin{aligned}
& -\int_{0}^{1}\left[\alpha_{0} u_{x} v_{x} \cos n \sin n+\frac{1}{2}\left(\alpha_{2}+\alpha_{3}+\alpha_{5}+\alpha_{6}\right) u_{x} v_{x} \cos n \sin n+\left(\alpha_{6}+\alpha_{8}\right) u_{x} v_{x} \cos n \sin n\right] \\
= & -\int_{0}^{1} u_{x} v_{x} \cos n \sin n\left[\alpha_{0}+\frac{1}{2}\left(\alpha_{2}+\alpha_{3}+\alpha_{5}+\alpha_{6}\right)+\left(\alpha_{6}+\alpha_{8}\right)\right] \\
= & -\int_{0}^{1}\left(\alpha_{0}+2 \alpha_{6}+\alpha_{8}\right) u_{x} v_{x} \cos n \sin n,
\end{aligned}
$$


where we have used $\alpha_{2}+\alpha_{3}=\alpha_{6}-\alpha_{5}$. The terms related to $u_{x}^{2}, v_{x}^{2}$ in (3.3) and (3.4) (without terms with $\alpha_{1}$ ) can be written as

$$
\begin{aligned}
& -\int_{0}^{1}\left[\left(\alpha_{0}+\alpha_{5}+\alpha_{6}+\alpha_{8}\right) u_{x}^{2} \cos ^{2} n+\left(\alpha_{4}+\alpha_{7}\right) u_{x}^{2}\right] \\
& -\int_{0}^{1}\left[\frac{1}{4}\left(2 \alpha_{4}-\alpha_{2}+\alpha_{5}+\alpha_{3}+\alpha_{6}\right) v_{x}^{2}-\frac{1}{2} \gamma_{2} v_{x}^{2} \cos (2 n)\right] .
\end{aligned}
$$

What left in (3.2)-(3.4) are all terms related to $u_{x} \dot{n}$ and $v_{x} \dot{n}$

$$
\begin{aligned}
& \int_{0}^{1}\left[\frac{1}{2} \gamma_{2} u_{x} \sin (2 n) \dot{n}+\left(\alpha_{2}+\alpha_{3}\right) u_{x} \dot{n} \cos n \sin n\right] \\
& +\int_{0}^{1}\left[\frac{1}{2}\left(\gamma_{1}-\gamma_{2} \cos (2 n)\right) v_{x} \dot{n}-\alpha_{2} v_{x} \dot{n} \cos ^{2} n+\alpha_{3} v_{x} \dot{n} \sin ^{2} n\right] \\
= & \int_{0}^{1} \gamma_{2} u_{x} \dot{n} \sin (2 n)+\int_{0}^{1}\left(\gamma_{1}-\gamma_{2} \cos (2 n)\right) v_{x} \dot{n},
\end{aligned}
$$

where we have used $\gamma_{1}=\alpha_{3}-\alpha_{2}$ and $\gamma_{2}=\alpha_{2}+\alpha_{3}=\alpha_{6}-\alpha_{5}$. Therefore, putting (3.5)-(3.8) into (3.2), we obtain

$$
\begin{aligned}
& \frac{1}{2} \frac{d}{d t} \int_{0}^{1} \rho\left(u^{2}+v^{2}\right)+\frac{1}{\gamma-1} \frac{d}{d t} \int_{0}^{1} \rho^{\gamma}+\frac{d}{d t} \frac{1}{2} \int_{0}^{1}\left(n_{x}\right)^{2} \\
= & -\alpha_{1} \int_{0}^{1}\left[u_{x} \cos ^{2} n+v_{x} \cos n \sin n\right]^{2}-\int_{0}^{1} u_{x} v_{x} \cos n \sin n\left(\alpha_{0}+2 \alpha_{6}+\alpha_{8}\right) \\
& -\int_{0}^{1}\left[\left(\alpha_{0}+\alpha_{5}+\alpha_{6}+\alpha_{8}\right) u_{x}^{2} \cos ^{2} n+\left(\alpha_{4}+\alpha_{7}\right) u_{x}^{2}\right] \\
& -\int_{0}^{1}\left[\frac{1}{4}\left(2 \alpha_{4}+\alpha_{5}+\alpha_{6}+\gamma_{1}\right) v_{x}^{2}-\frac{1}{2} \gamma_{2} v_{x}^{2} \cos (2 n)\right] \\
& -\gamma_{1} \int_{0}^{1} \dot{n}^{2}+\int_{0}^{1} \gamma_{2} u_{x} \dot{n} \sin (2 n)+\int_{0}^{1}\left(\gamma_{1}-\gamma_{2} \cos (2 n)\right) v_{x} \dot{n} .
\end{aligned}
$$

We first complete the square for all terms with $\dot{n}$ in (3.5)

$$
\begin{aligned}
& \gamma_{1} \int_{0}^{1} \dot{n}^{2}-\int_{0}^{1} \gamma_{2} u_{x} \dot{n} \sin (2 n)-\int_{0}^{1}\left(\gamma_{1}-\gamma_{2} \cos (2 n)\right) v_{x} \dot{n} \\
= & \gamma_{1} \int_{0}^{1} \dot{n}^{2}-2 \cdot \frac{1}{2} \int_{0}^{1} \sqrt{\gamma_{1}} \dot{n}\left(\frac{\gamma_{2}}{\sqrt{\gamma_{1}}} u_{x} \sin (2 n)+\frac{1}{\sqrt{\gamma_{1}}}\left(\gamma_{1}-\gamma_{2} \cos (2 n)\right) v_{x}\right) \\
= & \int_{0}^{1}\left[\sqrt{\gamma_{1}} \dot{n}-\frac{1}{2}\left(\frac{\gamma_{2}}{\sqrt{\gamma_{1}}} u_{x} \sin (2 n)+\frac{1}{\sqrt{\gamma_{1}}}\left(\gamma_{1}-\gamma_{2} \cos (2 n)\right) v_{x}\right)\right]^{2} \\
& -\frac{1}{4} \int_{0}^{1}\left(\frac{\gamma_{2}}{\sqrt{\gamma_{1}}} u_{x} \sin (2 n)+\frac{1}{\sqrt{\gamma_{1}}}\left(\gamma_{1}-\gamma_{2} \cos (2 n)\right) v_{x}\right)^{2} .
\end{aligned}
$$


The last term in (3.10) can also be rewritten as follows

$$
\begin{aligned}
& \left(\frac{\gamma_{2}}{\sqrt{\gamma_{1}}} u_{x} \sin (2 n)+\frac{1}{\sqrt{\gamma_{1}}}\left(\gamma_{1}-\gamma_{2} \cos (2 n)\right) v_{x}\right)^{2} \\
= & \frac{\gamma_{2}^{2}}{\gamma_{1}} u_{x}^{2} \sin ^{2}(2 n)+2 \frac{\gamma_{2}}{\gamma_{1}} u_{x} v_{x} \sin (2 n)\left(\gamma_{1}-\gamma_{2} \cos (2 n)\right)+\frac{1}{\gamma_{1}}\left(\gamma_{1}-\gamma_{2} \cos (2 n)\right)^{2} v_{x}^{2} \\
= & \frac{\gamma_{2}^{2}}{\gamma_{1}} u_{x}^{2} \sin ^{2}(2 n)+2 u_{x} v_{x} \sin (2 n)\left(\gamma_{2}-\frac{\gamma_{2}^{2}}{\gamma_{1}} \cos (2 n)\right) \\
& +\left(\gamma_{1}-2 \gamma_{2} \cos (2 n)+\frac{\gamma_{2}^{2}}{\gamma_{1}} \cos ^{2}(2 n)\right) v_{x}^{2} .
\end{aligned}
$$

To complete the square for the remaining terms, we first investigate the terms containing $u_{x} v_{x}$ in (3.10) and (3.11):

$$
\begin{aligned}
& \frac{1}{2} \alpha_{1} \int_{0}^{1} u_{x} v_{x} \sin (2 n)(1+\cos (2 n))+\frac{1}{2} \int_{0}^{1}\left(\alpha_{0}+2 \alpha_{6}+\alpha_{8}\right) u_{x} v_{x} \sin (2 n) \\
& -\frac{1}{2} \int_{0}^{1} u_{x} v_{x} \sin (2 n)\left(\gamma_{2}-\frac{\gamma_{2}^{2}}{\gamma_{1}} \cos (2 n)\right) \\
= & \frac{1}{2} \int_{0}^{1}\left(\alpha_{0}+\alpha_{1}+\alpha_{5}+\alpha_{6}+\alpha_{8}\right) u_{x} v_{x} \sin (2 n)+\frac{1}{2} \int_{0}^{1}\left(\alpha_{1}+\frac{\gamma_{2}^{2}}{\gamma_{1}}\right) u_{x} v_{x} \sin (2 n) \cos (2 n) \\
= & \int_{0}^{1}\left(\alpha_{0}+\alpha_{1}+\alpha_{5}+\alpha_{6}+\alpha_{8}\right) u_{x} v_{x} \sin n \cos n+\frac{1}{2} \int_{0}^{1}\left(\alpha_{1}+\frac{\gamma_{2}^{2}}{\gamma_{1}}\right) u_{x} v_{x} \sin (2 n) \cos (2 n) .
\end{aligned}
$$

Thus we can calculate the terms containing $u_{x}^{2}$ in (3.10) and (3.11) as follows

$$
\begin{aligned}
& \frac{1}{4} \int_{0}^{1}\left[\alpha_{1} u_{x}^{2}(1+\cos (2 n))^{2}-\frac{\gamma_{2}^{2}}{\gamma_{1}} u_{x}^{2} \sin ^{2}(2 n)\right] \\
& +\int_{0}^{1}\left[\left(\alpha_{0}+\alpha_{5}+\alpha_{6}+\alpha_{8}\right) u_{x}^{2} \cos ^{2} n+\left(\alpha_{4}+\alpha_{7}\right) u_{x}^{2}\right] \\
= & \frac{1}{4} \int_{0}^{1}\left[\alpha_{1} u_{x}^{2}\left(1+2 \cos (2 n)+\cos ^{2}(2 n)\right)-\frac{\gamma_{2}^{2}}{\gamma_{1}} u_{x}^{2}+\frac{\gamma_{2}^{2}}{\gamma_{1}} u_{x}^{2} \cos ^{2}(2 n)\right] \\
& +\int_{0}^{1}\left[\left(\alpha_{0}+\alpha_{5}+\alpha_{6}+\alpha_{8}\right) u_{x}^{2} \cos ^{2} n+2\left(\alpha_{4}+\alpha_{7}\right) u_{x}^{2}\right] \\
= & \frac{1}{4} \int_{0}^{1}\left(\alpha_{1}+\frac{\gamma_{2}^{2}}{\gamma_{1}}\right) u_{x}^{2} \cos ^{2}(2 n)+\int_{0}^{1}\left(\alpha_{0}+\alpha_{1}+\alpha_{5}+\alpha_{6}+\alpha_{8}\right) u_{x}^{2} \cos ^{2} n \\
& +\int_{0}^{1}\left[\frac{1}{4}\left(-\alpha_{1}-\frac{\gamma_{2}^{2}}{\gamma_{1}}\right) u_{x}^{2}+\left(\alpha_{4}+\alpha_{7}\right) u_{x}^{2}\right] .
\end{aligned}
$$


Similarly, the terms involving $v_{x}^{2}$ in (3.10) and (3.11) can be calculated as follows

$$
\begin{aligned}
& \frac{1}{4} \int_{0}^{1} \alpha_{1} v_{x}^{2} \sin ^{2}(2 n)+\int_{0}^{1}\left[\frac{1}{4}\left(2 \alpha_{4}+\alpha_{5}+\alpha_{6}+\gamma_{1}\right) v_{x}^{2}-\frac{1}{2} \gamma_{2} v_{x}^{2} \cos (2 n)\right] \\
& -\frac{1}{4} \int_{0}^{1}\left(\gamma_{1}-2 \gamma_{2} \cos (2 n)+\frac{\gamma_{2}^{2}}{\gamma_{1}} \cos ^{2}(2 n)\right) v_{x}^{2} \\
= & \frac{1}{4} \int_{0}^{1} \alpha_{1} v_{x}^{2} \sin ^{2}(2 n)+\frac{1}{4} \int_{0}^{1}\left(2 \alpha_{4}+\alpha_{5}+\alpha_{6}-\frac{\gamma_{2}^{2}}{\gamma_{1}} \cos ^{2}(2 n)\right) v_{x}^{2} \\
= & \frac{1}{8} \int_{0}^{1}\left(2 \alpha_{1}+3 \alpha_{4}+2 \alpha_{5}+2 \alpha_{6}\right) v_{x}^{2} \sin ^{2}(2 n)+\frac{1}{8} \int_{0}^{1} \alpha_{4} v_{x}^{2} \sin ^{2}(2 n) \\
& +\frac{1}{4} \int_{0}^{1}\left(2 \alpha_{4}+\alpha_{5}+\alpha_{6}-\frac{\gamma_{2}^{2}}{\gamma_{1}}\right) v_{x}^{2} \cos ^{2}(2 n) .
\end{aligned}
$$

For the terms with coefficient $\alpha_{1}+\frac{\gamma_{2}^{2}}{\gamma_{1}}$ in (3.12) and (3.13), we have

$$
\begin{aligned}
& \frac{1}{4} \int_{0}^{1}\left(\alpha_{1}+\frac{\gamma_{2}^{2}}{\gamma_{1}}\right) u_{x}^{2} \cos ^{2}(2 n)+\frac{1}{2} \int_{0}^{1}\left(\alpha_{1}+\frac{\gamma_{2}^{2}}{\gamma_{1}}\right) u_{x} v_{x} \sin (2 n) \cos (2 n) \\
= & \frac{1}{4}\left(\alpha_{1}+\frac{\gamma_{2}^{2}}{\gamma_{1}}\right) \int_{0}^{1}\left[\left(u_{x} \cos (2 n)+v_{x} \sin (2 n)\right)^{2}-v_{x}^{2} \sin ^{2}(2 n)\right] .
\end{aligned}
$$

The terms with coefficient $\alpha_{0}+\alpha_{1}+\alpha_{5}+\alpha_{6}+\alpha_{8}($ (3.12) and (3.13) can be written as

$$
\begin{aligned}
& \left(\alpha_{0}+\alpha_{1}+\alpha_{5}+\alpha_{6}+\alpha_{8}\right) \int_{0}^{1}\left(u_{x}^{2} \cos ^{2} n+u_{x} v_{x} \sin n \cos n\right) \\
= & \left(\alpha_{0}+\alpha_{1}+\alpha_{5}+\alpha_{6}+\alpha_{8}\right) \int_{0}^{1}\left[\left(u_{x} \cos n+\frac{1}{2} v_{x} \sin n\right)^{2}-\frac{1}{4} v_{x}^{2} \sin ^{2} n\right] .
\end{aligned}
$$

Collecting all the terms involving $v_{x}^{2}$ in (3.14)-(3.16), we have

$$
\begin{aligned}
& \frac{1}{8} \int_{0}^{1}\left(2 \alpha_{1}+3 \alpha_{4}+2 \alpha_{5}+2 \alpha_{6}\right) v_{x}^{2} \sin ^{2}(2 n)+\frac{1}{8} \int_{0}^{1} \alpha_{4} v_{x}^{2} \sin ^{2}(2 n) \\
& +\frac{1}{4} \int_{0}^{1}\left(2 \alpha_{4}+\alpha_{5}+\alpha_{6}-\frac{\gamma_{2}^{2}}{\gamma_{1}}\right) v_{x}^{2} \cos ^{2}(2 n)-\frac{1}{4}\left(\alpha_{1}+\frac{\gamma_{2}^{2}}{\gamma_{1}}\right) \int_{0}^{1} v_{x}^{2} \sin ^{2}(2 n) \\
& -\frac{1}{4}\left(\alpha_{0}+\alpha_{1}+\alpha_{5}+\alpha_{6}+\alpha_{8}\right) \int_{0}^{1} v_{x}^{2} \sin ^{2} n \\
= & \frac{1}{4} \int_{0}^{1}\left(2 \alpha_{4}+\alpha_{5}+\alpha_{6}-\frac{\gamma_{2}^{2}}{\gamma_{1}}\right) v_{x}^{2}-\frac{1}{4}\left(\alpha_{0}+\alpha_{1}+\alpha_{5}+\alpha_{6}+\alpha_{8}\right) \int_{0}^{1} v_{x}^{2} \sin ^{2} n .
\end{aligned}
$$


Therefore, putting the identities (3.10) (3.15)-(3.17) into (3.9) yields

$$
\begin{aligned}
& \frac{1}{2} \frac{d}{d t} \int_{0}^{1} \rho\left(u^{2}+v^{2}\right)+\frac{1}{\gamma-1} \frac{d}{d t} \int_{0}^{1} \rho^{\gamma}+\frac{d}{d t} \frac{1}{2} \int_{0}^{1}\left(n_{x}\right)^{2} \\
= & -\int_{0}^{1}\left[\sqrt{\gamma_{1}} \dot{n}-\frac{1}{2}\left(\frac{\gamma_{2}}{\sqrt{\gamma_{1}}} u_{x} \sin (2 n)+\frac{1}{\sqrt{\gamma_{1}}}\left(\gamma_{1}-\gamma_{2} \cos (2 n)\right) v_{x}\right)\right]^{2} \\
& -\int_{0}^{1}\left[\frac{1}{4}\left(-\alpha_{1}-\frac{\gamma_{2}^{2}}{\gamma_{1}}\right) u_{x}^{2}+\left(\alpha_{4}+\alpha_{7}\right) u_{x}^{2}\right]-\frac{1}{4} \int_{0}^{1}\left(2 \alpha_{4}+\alpha_{5}+\alpha_{6}-\frac{\gamma_{2}^{2}}{\gamma_{1}}\right) v_{x}^{2} \\
& -\frac{1}{4}\left(\alpha_{1}+\frac{\gamma_{2}^{2}}{\gamma_{1}}\right) \int_{0}^{1}\left(u_{x} \cos (2 n)+v_{x} \sin (2 n)\right)^{2} \\
& -\left(\alpha_{0}+\alpha_{1}+\alpha_{5}+\alpha_{6}+\alpha_{8}\right) \int_{0}^{1}\left[\left(u_{x} \cos n+\frac{1}{2} v_{x} \sin n\right)^{2}-\frac{1}{4} v_{x}^{2} \sin ^{2} n\right],
\end{aligned}
$$

which completes the proof of Lemma.

From the energy inequality above, we can obtain the following estimates for $n$.

Lemma 3.2 For any smooth solution to the system (1.7), it holds that

$$
\left\|n_{x x}\right\|_{L^{2}\left(0, T ; L^{2}\right)}+\left\|n_{t}\right\|_{L^{2}\left(0, T ; L^{2}\right)} \leq C\left(\mathcal{E}_{0}, T\right) .
$$

Proof. First notice that the equation of $n$ is

$$
\gamma_{1}\left(\dot{n}-\frac{1}{2} v_{x}\right)-\gamma_{2}\left(u_{x} \cos n \sin n+\frac{1}{2} v_{x}\left(1-2 \cos ^{2} n\right)\right)=n_{x x} .
$$

It is not hard to see that

$$
\begin{aligned}
& \gamma_{1}\left(\dot{n}-\frac{1}{2} v_{x}\right)-\gamma_{2}\left(u_{x} \cos n \sin n+\frac{1}{2} v_{x}\left(1-2 \cos ^{2} n\right)\right) \\
= & \gamma_{1} \dot{n}-\frac{1}{2} \gamma_{2} u_{x} \sin (2 n)-\frac{1}{2}\left(\gamma_{1}-\gamma_{2} \cos (2 n)\right) v_{x} .
\end{aligned}
$$

By the energy inequality, we obtain the estimates for $n_{x x}$. Next, by the equation of $n$ and the energy inequality, we obtain the estimate for $n_{t}$.

We also need to show the higher integrability of $\rho$, which is inspired by the argument in [4].

Lemma 3.3 For any smooth solution to the system (1.7), it holds that

$$
\|\rho\|_{L^{2 \gamma}([0,1] \times[0, T] ;)} \leq C\left(\mathcal{E}_{0}, T\right) .
$$

Proof. First set

$$
G(x, t):=\int_{0}^{x} \rho^{\gamma}-x \int_{0}^{1} \rho^{\gamma}
$$

It is easy to see that

$$
\frac{\partial G}{\partial x}=\rho^{\gamma}-\int_{0}^{1} \rho^{\gamma}, \quad G(0, t)=G(1, t)=0 .
$$

Notice that the equation of $u$ can be written as

$$
(\rho u)_{t}+\left(\rho u^{2}\right)_{x}+\left(\rho^{\gamma}\right)_{x}=J^{1}-\frac{1}{2}\left(\left(n_{x}\right)^{2}\right)_{x}
$$


where

$$
\begin{aligned}
J^{1}= & \left(\alpha_{0}+\alpha_{5}+\alpha_{6}+\alpha_{8}\right)\left(u_{x} \cos ^{2} n\right)_{x}+\alpha_{1}\left(u_{x} \cos ^{4} n\right)_{x}-\left(\alpha_{2}+\alpha_{3}\right)(\dot{n} \cos n \sin n)_{x}+\left(\alpha_{4}+\alpha_{7}\right) u_{x x} \\
& +\alpha_{0}\left(v_{x} \cos n \sin n\right)_{x}+\alpha_{1}\left(v_{x} \cos ^{3} n \sin n\right)_{x}+\frac{1}{2}\left(\alpha_{2}+\alpha_{3}+\alpha_{5}+\alpha_{6}\right)\left(v_{x} \cos n \sin n\right)_{x} .
\end{aligned}
$$

Multiplying this equation by $G(x, t)$, integrating over $[0,1] \times(0, T)$, and using integrating by parts, we obtain that

$$
\begin{aligned}
\int_{0}^{T} \int_{0}^{1} \rho^{2 \gamma}= & \int_{0}^{T}\left(\int_{0}^{1} \rho^{\gamma}\right)^{2}+\int_{0}^{T} \int_{0}^{1}(\rho u)_{t} G(x, t)-\int_{0}^{T} \int_{0}^{1} \rho u^{2} \frac{\partial G(x, t)}{\partial x} \\
& -\int_{0}^{T} \int_{0}^{1} J_{1} G(x, t)-\frac{1}{2} \int_{0}^{T} \int_{0}^{1}\left|n_{x}\right|^{2} \frac{\partial G(x, t)}{\partial x} \\
= & \sum_{i=1}^{5} I_{i} .
\end{aligned}
$$

For the first term, it is easy to estimate by energy inequality

$$
I_{1} \leq C\left(\mathcal{E}_{0}, T\right)
$$

For the second term, we need use integrating by parts with respect to $t$ to obtain

$$
\begin{aligned}
I_{2}= & \int_{0}^{1} \rho u G(x, T)-\int_{0}^{1} \rho u G(x, 0)-\int_{0}^{T} \int_{0}^{1} \rho u G_{t}(x, t) \\
& \leq C \sup _{0 \leq t \leq T}\left(\int_{0}^{1} \rho|u| \int_{0}^{1} \rho^{\gamma}\right)-\int_{0}^{T} \int_{0}^{1} \rho u G_{t}(x, t) \\
& \leq C \sup _{0 \leq t \leq T}\left(\int_{0}^{1} \rho|u|^{2} \int_{0}^{1} \rho^{\gamma}+\int_{0}^{1} \rho \int_{0}^{1} \rho^{\gamma}\right)-\int_{0}^{T} \int_{0}^{1} \rho u G_{t}(x, t) \\
& \leq C\left(\mathcal{E}_{0}, T\right)-\int_{0}^{T} \int_{0}^{1} \rho u G_{t}(x, t) .
\end{aligned}
$$

To estimate the last term here, we multiply the equation of $\rho$ by $\gamma \rho^{\gamma-1}$ to get

$$
\left(\rho^{\gamma}\right)_{t}+\left(\rho^{\gamma} u\right)_{x}+(\gamma-1) \rho^{\gamma} u_{x}=0
$$


Then it holds

$$
\begin{aligned}
& -\int_{0}^{T} \int_{0}^{1} \rho u G_{t}(x, t) \\
= & -\int_{0}^{T} \int_{0}^{1} \rho u\left(\int_{0}^{x} \rho_{t}^{\gamma}-x \int_{0}^{1} \rho_{t}^{\gamma}\right) \\
= & \int_{0}^{T} \int_{0}^{1} \rho u \int_{0}^{x}\left(\left(\rho^{\gamma} u\right)_{x}+(\gamma-1) \rho^{\gamma} u_{x}\right)-\int_{0}^{T} \int_{0}^{1} x \rho u \int_{0}^{1}\left(\left(\rho^{\gamma} u\right)_{x}+(\gamma-1) \rho^{\gamma} u_{x}\right) \\
= & \int_{0}^{T} \int_{0}^{1} \rho^{\gamma+1} u^{2}+(\gamma-1) \int_{0}^{T} \int_{0}^{1} \rho u\left(\int_{0}^{x} \rho^{\gamma} u_{x}-x \int_{0}^{1} \rho^{\gamma} u_{x}\right) \\
\leq & \int_{0}^{T} \int_{0}^{1} \rho^{\gamma+1} u^{2}+C \int_{0}^{T} \int_{0}^{1} \rho|u| \int_{0}^{1} \rho^{\gamma}\left|u_{x}\right| \\
\leq & \int_{0}^{T} \int_{0}^{1} \rho^{\gamma+1} u^{2}+C \int_{0}^{T}\left(\int_{0}^{1}\left(\rho+\rho|u|^{2}\right)\left(\int_{0}^{1} \rho^{2 \gamma}\right)^{\frac{1}{2}}\left(\int_{0}^{1}\left|u_{x}\right|^{2}\right)^{\frac{1}{2}}\right) \\
\leq & \int_{0}^{T} \int_{0}^{1} \rho^{\gamma+1} u^{2}+C\left(\mathcal{E}_{0}, T\right) \int_{0}^{T}\left(\left(\int_{0}^{1} \rho^{2 \gamma}\right)^{\frac{1}{2}}\left(\int_{0}^{1}\left|u_{x}\right|^{2}\right)^{\frac{1}{2}}\right) \\
\leq & \int_{0}^{T} \int_{0}^{1} \rho^{\gamma+1} u^{2}+\frac{1}{4} \int_{0}^{T} \int_{0}^{1} \rho^{2 \gamma}+C\left(\mathcal{E}_{0}, T\right) \int_{0}^{T} \int_{0}^{1}\left|u_{x}\right|^{2} \\
\leq & \int_{0}^{T} \int_{0}^{1} \rho^{\gamma+1} u^{2}+\frac{1}{4} \int_{0}^{T} \int_{0}^{1} \rho^{2 \gamma}+C\left(\mathcal{E}_{0}, T\right),
\end{aligned}
$$

where we have used the Cauchy inequality, the Hölder inequality, the Young inequality and the energy inequality. Hence we obtain

$$
I_{2} \leq \int_{0}^{T} \int_{0}^{1} \rho^{\gamma+1} u^{2}+\frac{1}{4} \int_{0}^{T} \int_{0}^{1} \rho^{2 \gamma}+C\left(\mathcal{E}_{0}, T\right) .
$$

For the third term in (3.21), it holds

$$
I_{3}=-\int_{0}^{T} \int_{0}^{1} \rho u^{2}\left(\rho^{\gamma}-\int_{0}^{1} \rho^{\gamma}\right)=-\int_{0}^{T} \int_{0}^{1} \rho^{\gamma+1} u^{2}+C\left(\mathcal{E}_{0}, T\right) .
$$

Then

$$
I_{2}+I_{3} \leq \frac{1}{4} \int_{0}^{T} \int_{0}^{1} \rho^{2 \gamma}+C\left(\mathcal{E}_{0}, T\right) .
$$

For the fourth term in (3.21), by integration by parts it holds

$$
\begin{aligned}
I_{4} \leq & \int_{0}^{T} \int_{0}^{1}\left(\left|u_{x}\right|+|\dot{n}|+\left|v_{x}\right|\right) \rho^{\gamma}+\int_{0}^{T} \int_{0}^{1}\left(\left|u_{x}\right|+\left|n_{t}\right|+\left|v_{x}\right|\right) \int_{0}^{1} \rho^{\gamma} \\
\leq & \frac{1}{4} \int_{0}^{T} \int_{0}^{1} \rho^{2 \gamma}+C \int_{0}^{T} \int_{0}^{1}\left(\left|u_{x}\right|^{2}+|\dot{n}|^{2}+\left|v_{x}\right|^{2}\right) \\
& +C\left(\mathcal{E}_{0}, T\right) \int_{0}^{T} \int_{0}^{1}\left(\left|u_{x}\right|^{2}+\left|n_{t}\right|^{2}+\left|v_{x}\right|^{2}\right)+C\left(\mathcal{E}_{0}, T\right) \\
\leq & \frac{1}{4} \int_{0}^{T} \int_{0}^{1} \rho^{2 \gamma}+C\left(\mathcal{E}_{0}, T\right) .
\end{aligned}
$$


For the last term in (3.21), it holds

$$
I_{5}=-\frac{1}{2} \int_{0}^{T} \int_{0}^{1}\left|n_{x}\right|^{2}\left(\rho^{\gamma}-\int_{0}^{1} \rho^{\gamma}\right) \leq C\left(\mathcal{E}_{0}, T\right) .
$$

Therefore, by adding all the estimates together in (3.21) we obtain

$$
\int_{0}^{T} \int_{0}^{1} \rho^{2 \gamma} \leq \frac{1}{2} \int_{0}^{T} \int_{0}^{1} \rho^{2 \gamma}+C\left(\mathcal{E}_{0}, T\right)
$$

which implies the estimate (3.20).

\section{Approximated solutions}

In this section, we first consider the case that the initial values are smooth enough, i.e. $\rho_{0} \in C^{1}$, $u_{0}, v_{0}, n_{0} \in C^{2}$, and $0<c_{0}^{-1} \leq \rho_{0} \leq c_{0}$ and $u_{0}=\frac{m_{0}}{\rho_{0}}, v_{0}=\frac{l_{0}}{\rho_{0}}$, and then construct the Galerkin approximation of $\rho, u, v$ and $n$.

Step 1. Recall that

$$
\phi_{j}(x)=\sin (j \pi x), \quad j=1,2, \ldots
$$

is an orthogonal base of $L^{2}(0,1)$. For any positive integer $k$, set

$$
\mathcal{X}_{k}=\operatorname{span}\left\{\phi_{1}, \phi_{2}, \cdots \phi_{k}\right\}
$$

and

$$
u_{0}^{k}=\sum_{j=0}^{k} \bar{c}_{j}^{k} \phi_{j}(x), \quad v_{0}^{k}=\sum_{j=0}^{k} \bar{d}_{j}^{k} \phi_{j}(x),
$$

for some constants

$$
\bar{c}_{j}^{k}=\int_{0}^{1} u_{0} \phi_{j}, \quad \bar{d}_{j}^{k}=\int_{0}^{1} v_{0} \phi_{j} .
$$

Then $\left(u_{0}^{k}, v_{0}^{k}\right) \rightarrow\left(u_{0}, v_{0}\right)$ in $C^{2}$ as $k \rightarrow \infty$. Let

$$
u_{k}=\sum_{j=0}^{k} c_{j}^{k}(t) \phi_{j}(x), \quad v_{k}=\sum_{j=0}^{k} d_{j}^{k}(t) \phi_{j}(x)
$$

be the finite dimensional approximation of $u$, and $v$, and we want to solve the approximation system:

$$
\left\{\begin{array}{l}
\left(\rho_{k}\right)_{t}+\left(\rho_{k} u_{k}\right)_{x}=0, \\
\rho_{k}\left(u_{k}\right)_{t}+\rho_{k} u_{k}\left(u_{k}\right)_{x}+\left(\rho_{k}^{\gamma}\right)_{x}=J_{k}^{1}-\left(n_{k}\right)_{x x}\left(n_{k}\right)_{x}, \\
\rho_{k}\left(v_{k}\right)_{t}+\rho_{k} u_{k}\left(v_{k}\right)_{x}=J_{k}^{2}, \\
\gamma_{1}\left(\dot{n}_{k}-\frac{1}{2}\left(v_{k}\right)_{x}\right)-\gamma_{2}\left(\left(u_{k}\right)_{x} \cos n_{k} \sin n_{k}+\frac{1}{2}\left(v_{k}\right)_{x}\left(1-2 \cos ^{2} n_{k}\right)\right)=\left(n_{k}\right)_{x x} .
\end{array}\right.
$$


Here $J_{k}^{1}, J_{k}^{2}$ have the same form as $J^{1}, J^{2}$, but with $u, v$ replaced by $u_{k}, v_{k}$. For this system, we consider the following initial and boundary values

$$
\begin{gathered}
\left(\rho_{k}, u_{k}, v_{k}, n_{k}\right)(x, 0)=\left(\rho_{0}, u_{0}^{k}, v_{0}^{k}, n_{0}\right)(x), \\
u_{k}(0, t)=v_{k}(0, t)=u_{k}(1, t)=v_{k}(1, t)=0, \quad\left(n_{k}\right)_{x}(0, t)=\left(n_{k}\right)_{x}(1, t)=0 .
\end{gathered}
$$

Step 2. The first step is to solve $\rho_{k}$ and $n_{k}$ by assuming $u_{k}, v_{k} \in C^{0}\left(0, T ; C^{2}\right)$ for a fixed $k$. To this end, we rewrite the equations of $\rho_{k}$ and $n_{k}$ in the Lagrange coordinate system.

Without loss of generality, in this section, we assume that

$$
\int_{0}^{1} \rho_{0}(x) d x=1
$$

For any $T>0$, we introduce the Lagrangian coordinate $(X, \tau) \in(0,1) \times[0, T)$ by

$$
X(x, t)=\int_{0}^{x} \rho_{k}(y, t) d y, \quad \tau(x, t)=t
$$

If $\rho_{k}(x, t) \in C^{1}((0,1) \times[0, T))$ is positive and $\int_{0}^{1} \rho_{k}(x, t) d x=1$ for all $t \in[0, T)$, then the map $(x, t) \rightarrow(X, \tau):(0,1) \times(0, T) \rightarrow(0,1) \times(0, T)$ is a $C^{1}$-bijection such that $X(0, t)=0, X(1, t)=1$. By the chain rule, we have

$$
\frac{\partial}{\partial t}=-\rho_{k} u_{k} \frac{\partial}{\partial X}+\frac{\partial}{\partial \tau}, \quad \frac{\partial}{\partial x}=\rho_{k} \frac{\partial}{\partial X}
$$

The equation of $\rho_{k}$ can be rewritten as

$$
\left(\rho_{k}\right)_{\tau}+\rho_{k}^{2}\left(u_{k}\right)_{X}=0
$$

along with the initial condition

$$
\rho_{k}(X, 0)=\rho_{0} .
$$

Suppose $u_{k} \in C^{0}\left(0, T ; C^{2}\right)$ with $\left\|u_{k}\right\|_{C^{0}\left(0, T ; C^{2}\right)} \leq M_{0}$. Then $\rho_{k}$ can be solved explicitly by

$$
\rho_{k}(X, \tau)=\frac{\rho_{0}(X)}{1+\rho_{0}(X) \int_{0}^{\tau}\left(u_{k}\right)_{X}(X, s) d s} .
$$

Hence, for any $T \leq \frac{1}{2 c_{0} M_{0}}$, we have

$$
\begin{gathered}
\rho_{k}(X, \tau) \leq \frac{\rho_{0}(X)}{1-\left|\rho_{0}(X) \int_{0}^{\tau}\left(u_{k}\right)_{X}(X, s) d s\right|} \leq \frac{c_{0}}{1-c_{0} M_{0} T} \leq 2 c_{0}, \\
\rho_{k}(X, \tau) \geq \frac{\rho_{0}(X)}{1+\left|\rho_{0}(X) \int_{0}^{\tau}\left(u_{k}\right)_{X}(X, s) d s\right|} \geq \frac{c_{0}^{-1}}{1+c_{0} M_{0} T} \geq \frac{c_{0}^{-1}}{2} .
\end{gathered}
$$

Similarly, since $\rho_{0} \in C^{1}, u_{k} \in C^{0}\left(0, T ; C^{2}\right)$, we conclude that for sufficiently small $T\left(c_{0}, M_{0}\right)>0$,

$$
\left\|\rho_{k}\right\|_{C^{0}\left(0, T ; C^{1}\right)}+\left\|\left(\rho_{k}\right)_{t}\right\|_{C^{0}((0,1) \times(0, T))} \leq M_{1}
$$


for some positive constant $M_{1}$.

Furthermore, suppose that $\rho_{k}^{1}, \rho_{k}^{2}$ are solutions of equation (4.5) corresponding to $u_{k}^{1}, u_{k}^{2} \in$ $C^{0}\left(0, T ; C^{2}\right)$, with the same initial condition, we can conclude from (4.5) that

$$
\left(\frac{1}{\rho_{k}^{1}}-\frac{1}{\rho_{k}^{2}}\right)_{\tau}=\left(u_{k}^{1}-u_{k}^{2}\right)_{X}
$$

Integrating with respect to $\tau$, we obtain

$$
\rho_{k}^{1}-\rho_{k}^{2}=\rho_{k}^{1} \rho_{k}^{2} \int_{0}^{\tau}\left(u_{k}^{1}-u_{k}^{2}\right)_{X}
$$

which, combined with (4.10), implies that

$$
\left\|\rho_{k}^{1}-\rho_{k}^{2}\right\|_{C^{0}\left(0, T ; C^{1}\right)} \leq C\left(M_{1}, T\right) T\left\|u_{k}^{1}-u_{k}^{2}\right\|_{C^{0}\left(0, T ; C^{2}\right)} .
$$

Step 3. Similarly, we can rewrite the equation of $n$ in the Lagrange coordinate as

$$
\gamma_{1}\left(\left(n_{k}\right)_{\tau}-\frac{1}{2} \rho_{k}\left(v_{k}\right)_{X}\right)-\frac{\gamma_{2}}{2}\left(\rho_{k}\left(u_{k}\right)_{X} \sin \left(2 n_{k}\right)-\rho_{k}\left(v_{k}\right)_{X} \cos \left(2 n_{k}\right)\right)=\rho_{k}\left(\rho_{k}\left(n_{k}\right)_{X}\right)_{X} .
$$

For this system, we consider the following initial and boundary values

$$
\begin{gathered}
n_{k}(X, 0)=n_{0}(X) \\
\left(n_{k}\right)_{X}(0, \tau)=\left(n_{k}\right)_{X}(1, \tau)=0 .
\end{gathered}
$$

By the standard Schauder theory of parabolic equations, we conclude that

$$
\left\|n_{k}\right\|_{C^{1}\left(0, T ; C^{2}\right)} \leq C\left\|n_{0}\right\|_{C^{2}}+C\left\|\rho_{k}\left(v_{k}\right)_{X}\right\|_{C^{0}((0,1) \times(0, T))}+C\left\|\rho_{k}\left(u_{k}\right)_{X}\right\|_{C^{0}((0,1) \times(0, T))} \leq M_{2},
$$

for some positive constant $M_{2}$.

Furthermore, suppose that $n_{k}^{1}, n_{k}^{2}$ are solutions of equation (4.12) corresponding to $\rho_{k}^{1}, \rho_{k}^{2} \in$ $C^{1}((0,1) \times(0, T))$ and $u_{k}^{1}, u_{k}^{2} \in C^{0}\left(0, T ; C^{2}\right)$, subject to the same initial condition. Denote

$$
\bar{n}_{k}=n_{k}^{1}-n_{k}^{2}, \quad \bar{\rho}_{k}=\rho_{k}^{1}-\rho_{k}^{2}, \quad \bar{u}_{k}=u_{k}^{1}-u_{k}^{2} .
$$

Then from (4.12) we have that

$$
\begin{aligned}
& \gamma_{1}\left(\bar{n}_{k}\right)_{\tau}-\left(\rho_{k}^{1}\right)^{2}\left(\bar{n}_{k}\right)_{X X} \\
& =\bar{\rho}_{k}\left(\rho_{k}^{1}+\rho_{k}^{2}\right)\left(n_{k}^{2}\right)_{X X}+\bar{\rho}_{k}\left(\rho_{k}^{1}\right)_{X}\left(n_{k}^{1}\right)_{X}+\rho_{k}^{2}\left(\bar{\rho}_{k}\right)_{X}\left(n_{k}^{1}\right)_{X}+\rho_{k}^{2}\left(\rho_{k}^{2}\right)_{X}\left(\bar{n}_{k}\right)_{X} \\
& +\frac{\gamma_{1}}{2}\left(\bar{\rho}_{k}\left(v_{k}^{1}\right)_{X}+\rho_{k}^{2}\left(\bar{v}_{k}^{1}\right)_{X}\right) \\
& -\frac{\gamma_{2}}{2}\left(\bar{\rho}_{k}\left(v_{k}^{1}\right)_{X} \cos \left(2 n_{k}^{1}\right)+\rho_{k}^{2}\left(\bar{v}_{k}\right)_{X} \cos \left(2 n_{k}^{1}\right)-2 \rho_{k}^{2}\left(v_{k}^{2}\right)_{X} \sin \left(\bar{n}_{k}\right) \sin \left(n_{k}^{1}+n_{k}^{2}\right)\right) \\
& +\frac{\gamma_{2}}{2}\left(\bar{\rho}_{k}\left(u_{k}^{1}\right)_{X} \sin \left(2 n_{k}^{1}\right)+\rho_{k}^{2}\left(\bar{u}_{k}\right)_{X} \sin \left(2 n_{k}^{1}\right)+2 \rho_{k}^{2}\left(u_{k}^{2}\right)_{X} \sin \left(\bar{n}_{k}\right) \cos \left(n_{k}^{1}+n_{k}^{2}\right)\right) .
\end{aligned}
$$


By the standard $W_{2}^{2,1}$-estimate of parabolic equations, we conclude that

$$
\begin{aligned}
& \left\|\bar{n}_{k}\right\|_{W_{2}^{2,1}([0,1] \times(0, T))} \\
& \leq C\left\|\bar{\rho}_{k}\right\|_{L^{2}\left(0, T ; H^{1}\right)}+C\left\|\bar{n}_{k}\right\|_{L^{2}\left(0, T ; L^{2}\right)}+C\left\|\bar{v}_{k}\right\|_{L^{2}\left(0, T ; H^{1}\right)}+C\left\|\bar{u}_{k}\right\|_{L^{2}\left(0, T ; H^{1}\right)} \\
& \leq C T^{\frac{1}{2}}\left\|\bar{\rho}_{k}\right\|_{C^{0}\left(0, T ; C^{1}\right)}+C\left\|\bar{n}_{k}\right\|_{L^{2}\left(0, T ; L^{2}\right)}+C T^{\frac{1}{2}}\left\|\bar{v}_{k}\right\|_{C^{0}\left(0, T ; C^{1}\right)}+C T^{\frac{1}{2}}\left\|\bar{u}_{k}\right\|_{C^{0}\left(0, T ; C^{1}\right)} \\
& \leq C T^{\frac{1}{2}}\left\|\bar{u}_{k}\right\|_{C^{0}\left(0, T ; C^{2}\right)}+C T^{\frac{1}{2}}\left\|\bar{v}_{k}\right\|_{C^{0}\left(0, T ; C^{1}\right)}+C\left\|\bar{n}_{k}\right\|_{L^{2}\left(0, T ; L^{2}\right)} .
\end{aligned}
$$

Since $\bar{n}_{k}(\tau, 0)=0$, we obtain that

$$
\left\|\bar{n}_{k}\right\|_{L^{2}\left(0, T ; L^{2}\right)} \leq C T\left\|\bar{n}_{k}\right\|_{W_{2}^{2,1}([0,1] \times(0, T))} .
$$

If we choose $T>0$ small enough, we obtain

$$
\left\|\bar{n}_{k}\right\|_{W_{2}^{2,1}([0,1] \times(0, T))} \leq C\left(M_{1}, M_{2}, T\right) T^{\frac{1}{2}}\left(\left\|\bar{u}_{k}\right\|_{C^{0}\left(0, T ; C^{2}\right)}+\left\|\bar{v}_{k}\right\|_{C^{0}\left(0, T ; C^{1}\right)}\right) .
$$

Step 4. To obtain the estimates for $u_{k}$ and $v_{k}$, first notice that the equation of $u_{k}$ and $v_{k}$ can be understood in the weak senses, i.e., for any $\phi(x) \in \mathcal{X}_{k}$ and $t \in[0, T]$, it holds

$$
\begin{gathered}
\int_{0}^{1} \rho_{k} u_{k} \phi-\int \rho_{0} u_{0}^{k} \phi=\int_{0}^{t} \int_{0}^{1} \mathcal{P}^{1}\left(\rho_{k}, u_{k}, v_{k}, n_{k}\right) \phi+\left(\alpha_{2}+\alpha_{3}\right) \int_{0}^{t} \int_{0}^{1} \dot{n} \cos n \sin n \phi_{x} \\
\int_{0}^{1} \rho_{k} v_{k} \phi-\int \rho_{0} v_{0}^{k} \phi=\int_{0}^{t} \int_{0}^{1} \mathcal{P}^{2}\left(\rho_{k}, u_{k}, v_{k}, n_{k}\right) \phi-\int_{0}^{t} \int_{0}^{1}\left(\alpha_{2} \dot{n}_{k} \cos ^{2} n_{k}-\alpha_{3} \dot{n}_{k} \sin ^{2} n_{k}\right) \phi_{x}
\end{gathered}
$$

where

$$
\begin{aligned}
& \mathcal{P}^{1}\left(\rho_{k}, u_{k}, v_{k}, n_{k}\right) \\
= & \left(\alpha_{0}+\alpha_{5}+\alpha_{6}+\alpha_{8}\right)\left(\left(u_{k}\right)_{x} \cos ^{2} n_{k}\right)_{x}+\alpha_{1}\left(\left(u_{k}\right)_{x} \cos ^{4} n_{k}\right)_{x}+\left(\alpha_{4}+\alpha_{7}\right)\left(u_{k}\right)_{x x} \\
& +\alpha_{0}\left(\left(v_{k}\right)_{x} \cos n_{k} \sin n_{k}\right)_{x}+\alpha_{1}\left(\left(v_{k}\right)_{x} \cos ^{3} n_{k} \sin n_{k}\right)_{x} \\
& +\frac{1}{2}\left(\alpha_{2}+\alpha_{3}+\alpha_{5}+\alpha_{6}\right)\left(\left(v_{k}\right)_{x} \cos n_{k} \sin n_{k}\right)_{x}-\left(\rho_{k} u_{k} u_{k}\right)_{x}-\left(\rho_{k}^{\gamma}\right)_{x}-\left(n_{k}\right)_{x x}\left(n_{k}\right)_{x},
\end{aligned}
$$

and

$$
\begin{aligned}
\mathcal{P}^{2}\left(\rho_{k}, u_{k}, v_{k}, n_{k}\right) & =\alpha_{1}\left(\left(v_{k}\right)_{x} \cos ^{2} n_{k} \sin ^{2} n_{k}\right)_{x}+\frac{1}{2}\left(-\alpha_{2}+\alpha_{5}\right)\left(\left(v_{k}\right)_{x} \cos ^{2} n_{k}\right)_{x} \\
& +\frac{1}{2}\left(\alpha_{3}+\alpha_{6}\right)\left(\left(v_{k}\right)_{x} \sin ^{2} n_{k}\right)_{x}+\frac{1}{2} \alpha_{4}\left(v_{k}\right)_{x x} \\
& +\alpha_{1}\left(\left(u_{k}\right)_{x} \cos ^{3} n_{k} \sin n_{k}\right)_{x}+\left(\alpha_{6}+\alpha_{8}\right)\left(\left(u_{k}\right)_{x} \cos n_{k} \sin n_{k}\right)_{x}-\left(\rho_{k} v_{k} v_{k}\right)_{x}
\end{aligned}
$$

Similarly to the energy inequality (1.10), we can obtain the same form of energy estimates for the system (4.1) so that

$$
\left\|u_{k}\right\|_{C^{0}\left(0, T ; C^{2}\right)}+\left\|v_{k}\right\|_{C^{0}\left(0, T ; C^{2}\right)} \leq C\left\|u_{k}\right\|_{C^{0}\left(0, T ; L^{2}\right)}+C\left\|v_{k}\right\|_{C^{0}\left(0, T ; L^{2}\right)} \leq M_{3},
$$

provided $\inf _{(x, t)} \rho_{k}(x, t)>0$. Here we have used the fact that the dimension of $\mathcal{X}_{k}$ is finite. 
To apply the contraction map theorem, we define the linear operator $\mathcal{N}\left[\rho_{k}\right]: \mathcal{X}_{k} \rightarrow \mathcal{X}_{k}^{*}$ by

$$
\left\langle\mathcal{N}\left[\rho_{k}\right] \psi, \phi\right\rangle=\int_{0}^{1} \rho_{k} \psi \phi, \psi, \phi \in \mathcal{X}_{k}
$$

It is easy to see that

$$
\left\|\mathcal{N}\left[\rho_{k}\right]\right\|_{\mathcal{L}\left(\mathcal{X}_{k}, \mathcal{X}_{k}^{*}\right)} \leq C(k)\left\|\rho_{k}\right\|_{L^{1}}
$$

If $\inf _{x} \rho_{k}>0$, the operator $\mathcal{N}\left[\rho_{k}\right]$ is invertible and

$$
\left\|\mathcal{N}^{-1}\left[\rho_{k}\right]\right\|_{\mathcal{L}\left(\mathcal{X}_{k}^{*}, \mathcal{X}_{k}\right)} \leq\left(\inf _{x} \rho_{k}\right)^{-1}
$$

Furthermore, for any $\rho_{k}^{i} \in L^{1}$ and $\inf _{x} \rho_{k}^{i}>0, i=1,2$, it is easy to see that

$$
\mathcal{N}^{-1}\left[\rho_{k}^{1}\right]-\mathcal{N}^{-1}\left[\rho_{k}^{2}\right]=\mathcal{N}^{-1}\left[\rho_{k}^{2}\right]\left(\mathcal{N}\left[\rho_{k}^{2}\right]-\mathcal{N}\left[\rho_{k}^{1}\right]\right) \mathcal{N}^{-1}\left[\rho_{k}^{1}\right]
$$

which implies that

$$
\left\|\mathcal{N}^{-1}\left[\rho_{k}^{1}\right]-\mathcal{N}^{-1}\left[\rho_{k}^{2}\right]\right\|_{\mathcal{L}\left(\mathcal{X}_{k}^{*}, \mathcal{X}_{k}\right)} \leq C\left\|\mathcal{N}\left[\rho_{k}^{1}\right]-\mathcal{N}\left[\rho_{k}^{2}\right]\right\|_{\mathcal{L}\left(\mathcal{X}_{k}, \mathcal{X}_{k}^{*}\right)} \leq C\left\|\rho_{k}^{1}-\rho_{k}^{2}\right\|_{L^{1}}
$$

Hence by the estimates (4.11), (4.16) and (4.20), we can apply the standard contraction map theorem to obtain the local existence of a unique solution $u_{k}, v_{k} \in C\left(0, T_{k} ; \mathcal{X}_{k}\right)$ to (4.17) and (4.18) for some $T_{k}>0$. Then by the equations (4.5) and (4.12), we can solve for $\rho_{k}, n_{k}$, which provides a unique local solution to the approximated system (4.1) for any fixed $k$.

Step 5. In this step, we will establish a uniform estimate of the local solution until $T_{k}$ in order to extend the solution beyond $T_{k}$ to any time $T>0$, which implies the existence of unique global solution of the system (4.1) for any fixed $k$. We first show the following uniform estimate for $\rho_{k}$

Claim: For any $x \in[0,1]$ and $t \in\left[0, T_{k}\right]$, it holds

$$
\frac{1}{c_{1} e^{t}} \leq \rho_{k}(x, t) \leq c_{1} e^{t}
$$

for some constant $c_{1}>0$.

Indeed, similar to the energy inequality (1.10), we can obtain the same form of energy estimate for system (4.1) so that

$$
\begin{aligned}
& \left\|\left(u_{k}\right)_{x}\right\|_{L^{2}\left(0, T_{k} ; H^{2}\right)}+\left\|\left(v_{k}\right)_{x}\right\|_{L^{2}\left(0, T_{k} ; H^{2}\right)} \\
\leq & \left.C\left\|\left(u_{k}\right)_{x}\right\|_{L^{2}\left((0,1) \times\left(0, T_{k}\right)\right.}\right)+C\left\|\left(v_{k}\right)_{x}\right\|_{L^{2}\left((0,1) \times\left(0, T_{k}\right)\right)} \leq M_{4} .
\end{aligned}
$$

By the first equation of (4.1), we can find $x_{0}(t) \in(0,1)$ such that

$$
\rho_{k}\left(x_{0}(t), t\right)=\int_{0}^{1} \rho_{k}=\int_{0}^{1} \rho_{0}=1 .
$$

Then

$$
\frac{1}{\rho_{k}(x, t)}=\frac{1}{\rho_{k}\left(x_{0}(t), t\right)}+\int_{x_{0}(t)}^{x}\left(\frac{1}{\rho_{k}}\right)_{y} \leq 1+\frac{1}{2}\left\|\frac{1}{\rho_{k}(x, t)}\right\|_{L^{\infty}}+\frac{1}{2} \int_{0}^{1} \rho_{k}\left|\left(\frac{1}{\rho_{k}}\right)_{x}\right|^{2}
$$


which implies

$$
\left\|\frac{1}{\rho_{k}(x, t)}\right\|_{L^{\infty}} \leq 2+\int_{0}^{1} \rho_{k}\left|\left(\frac{1}{\rho_{k}}\right)_{x}\right|^{2} .
$$

By the first equation of (4.1), we have

$$
\begin{aligned}
& \frac{d}{d t} \int_{0}^{1} \rho_{k}\left|\left(\frac{1}{\rho_{k}}\right)_{x}\right|^{2} \\
= & \int_{0}^{1}\left(\rho_{k}\right)_{t}\left|\left(\frac{1}{\rho_{k}}\right)_{x}\right|^{2}+2 \int_{0}^{1} \rho_{k}\left(\frac{1}{\rho_{k}}\right)_{x}\left(\frac{1}{\rho_{k}}\right)_{x t} \\
= & -\int_{0}^{1}\left(\rho_{k} u_{k}\right)_{x}\left|\left(\frac{1}{\rho_{k}}\right)_{x}\right|^{2}+2 \int_{0}^{1} \rho_{k}\left(\frac{1}{\rho_{k}}\right)_{x}\left(\frac{\left(\rho_{k} u_{k}\right)_{x}}{\rho_{k}^{2}}\right)_{x} .
\end{aligned}
$$

The last term on the right hand side can be computed by

$$
\begin{aligned}
& 2 \int_{0}^{1} \rho_{k}\left(\frac{1}{\rho_{k}}\right)_{x}\left(\frac{\left(\rho_{k} u_{k}\right)_{x}}{\rho_{k}^{2}}\right)_{x} \\
= & 2 \int_{0}^{1} \rho_{k}\left(\frac{1}{\rho_{k}}\right)_{x}\left[\left(\left(-\frac{1}{\rho_{k}}\right)_{x} u_{k}\right)_{x}+\left(\frac{\left(u_{k}\right)_{x}}{\rho_{k}}\right)_{x}\right] \\
= & -\int_{0}^{1} \rho_{k} u_{k} \frac{\partial}{\partial x}\left|\left(\frac{1}{\rho_{k}}\right)_{x}\right|^{2}+2 \int_{0}^{1}\left(\frac{1}{\rho_{k}}\right)_{x}\left(u_{k}\right)_{x x} .
\end{aligned}
$$

Combining (4.25) with (4.24), we conclude that

$$
\frac{d}{d t} \int_{0}^{1} \rho_{k}\left|\left(\frac{1}{\rho_{k}}\right)_{x}\right|^{2}=2 \int_{0}^{1}\left(\frac{1}{\rho_{k}}\right)_{x}\left(u_{k}\right)_{x x} .
$$

The right hand side can be estimated as follows

$$
\begin{aligned}
& \left|\int_{0}^{1}\left(\frac{1}{\rho_{k}}\right)_{x}\left(u_{k}\right)_{x x}\right| \\
\leq & \int_{0}^{1} \rho_{k}^{\frac{1}{2}}\left|\left(\frac{1}{\rho_{k}}\right)_{x}\right| \rho_{k}^{-\frac{1}{2}}\left|\left(u_{k}\right)_{x x}\right| \\
\leq & \frac{1}{2} \int_{0}^{1} \rho_{k}\left|\left(\frac{1}{\rho_{k}}\right)_{x}\right|^{2}+\left.\frac{1}{2}|| \frac{1}{\rho_{k}(x, t)}\right|_{L^{\infty}} \int_{0}^{1}\left|\left(u_{k}\right)_{x x}\right|^{2} \\
\leq & \frac{1}{2}\left(1+\int_{0}^{1}\left|\left(u_{k}\right)_{x x}\right|^{2}\right) \int_{0}^{1} \rho_{k}\left|\left(\frac{1}{\rho_{k}}\right)_{x}\right|^{2}+\int_{0}^{1}\left|\left(u_{k}\right)_{x x}\right|^{2} .
\end{aligned}
$$

where we have used (4.23) in last inequality. Denote

$$
\mathcal{Q}\left(\rho_{k}\right)=\int_{0}^{1} \rho_{k}\left|\left(\frac{1}{\rho_{k}}\right)_{x}\right|^{2}, \quad a(t)=1+\int_{0}^{1}\left|\left(u_{k}\right)_{x x}\right|^{2} .
$$

Then by (4.25), we have

$$
\frac{d}{d t} \mathcal{Q}\left(\rho_{k}\right) \leq a(t) \mathcal{Q}\left(\rho_{k}\right)+\int_{0}^{1}\left|\left(u_{k}\right)_{x x}\right|^{2}
$$


which is equivalent to

$$
\mathcal{Q}\left(\rho_{k}\right)-\mathcal{Q}\left(\rho_{0}\right) \leq 2 \int_{0}^{t} \int_{0}^{1}\left|\left(u_{k}\right)_{x x}\right|^{2}+\int_{0}^{t} a(t) \mathcal{Q}\left(\rho_{k}\right) \leq 2 M_{4}+\int_{0}^{t} a(t) \mathcal{Q}\left(\rho_{k}\right),
$$

where we have used (4.22) in last step. By the Gronwall inequality, we obtain

$$
\mathcal{Q}\left(\rho_{k}\right) \leq\left(\mathcal{Q}\left(\rho_{0}\right)+2 M_{4}\right) \exp \left(\int_{0}^{t} a(s)\right) \leq\left(\mathcal{Q}\left(\rho_{0}\right)+2 M_{4}\right) \exp \left(t+M_{4}\right) \leq C e^{t} .
$$

Combining (4.23) and (4.27) together, we can prove the left hand side of (4.21).

Denote $\gamma=1+2 \delta$ for some $\delta>0$. Then it holds

$$
\left\|\rho_{k}^{\delta}\right\|_{L^{\infty}} \leq \int_{0}^{1} \rho_{k}^{\delta}+\delta \int_{0}^{1} \rho_{k}^{\delta-1}\left(\rho_{k}\right)_{x} \leq\left(\int_{0}^{1} \rho_{k}^{\gamma}\right)^{\frac{\delta}{\gamma}}+C\left(\int_{0}^{1} \rho_{k}^{\gamma}\right)^{\frac{1}{2}}\left(\mathcal{Q}\left(\rho_{k}\right)\right)^{\frac{1}{2}} \leq C e^{t},
$$

which completes the proof of the Claim.

By using the uniform estimate (4.21) for $\rho_{k}$ and the energy inequality, we can show

$$
\left\|u_{k}\right\|_{C^{0}\left(0, T_{k} ; \mathcal{X}_{k}\right)}+\left\|v_{k}\right\|_{C^{0}\left(0, T_{k} ; \mathcal{X}_{k}\right)} \leq C\left\|u_{k}\right\|_{C^{0}\left(0, T_{k} ; L^{2}\right)}+C\left\|v_{k}\right\|_{C^{0}\left(0, T_{k} ; L^{2}\right)} \leq M_{5} .
$$

Therefore, we can extend the solution beyond $T_{k}$ to any time $T>0$, which implies the existence of a unique smooth solution of the system (4.1) for any fixed $k$.

\section{$5 \quad$ Existence of global weak solutions}

Step 1. Taking $k \rightarrow \infty$ in the approximated system (4.1), we may obtain the existence of a global weak solution with a smooth initial and boundary value and $\rho_{0}>\delta>0$. Since the limit process of this step is similar to the next step when $\delta \rightarrow 0$, we omit the details of this step.

Step 2. We first approximate the general initial and boundary data in Theorem 1.2 by smooth functions. We may extend $n$ to $\tilde{n}_{0} \in H^{1}(\mathbb{R})$ such that $n_{0}=\tilde{n}_{0}$ on $(0,1)$, and obtain the smooth approximation of initial data by the standard mollification as follows

$$
\rho_{0}^{\delta}=\eta_{\delta} * \hat{\rho}_{0}+\delta, \quad u_{0}^{\delta}=\frac{1}{\sqrt{\rho_{0}^{\delta}}} \eta_{\delta} *\left(\frac{\widehat{m_{0}}}{\sqrt{\rho_{0}}}\right), \quad v_{0}^{\delta}=\frac{1}{\sqrt{\rho_{0}^{\delta}}} \eta_{\delta} *\left(\frac{\widehat{l_{0}}}{\sqrt{\rho_{0}}}\right), \quad n_{0}^{\delta}=\frac{\eta_{\delta} * \tilde{n}_{0}}{\left|\eta_{\delta} * \tilde{n}_{0}\right|}
$$

where, for small $\delta>0, \eta_{\delta}=\frac{1}{\delta} \eta(\dot{\bar{\delta}})$ is the standard mollifier, $\hat{f}$ is the zero extension of $f$ from $(0,1)$ to $\mathbb{R}$. Therefore $\rho_{0}^{\delta}, u_{0}^{\delta}, v_{0}^{\delta}, n_{0}^{\delta} \in C^{2+\alpha}([0,1])$ for $0<\alpha<1$, and it holds

$$
\begin{gathered}
\rho_{0}^{\delta} \geq \delta>0, \quad \rho_{0}^{\delta} \rightarrow \rho_{0} \text { in } L^{\gamma}, \quad n_{0}^{\delta} \rightarrow n_{0} \text { in } H^{1}, \\
\sqrt{\rho_{0}^{\delta}} u_{0}^{\delta} \rightarrow \frac{m_{0}}{\sqrt{\rho_{0}}} \text { in } L^{2}, \quad \sqrt{\rho_{0}^{\delta}} v_{0}^{\delta} \rightarrow \frac{l_{0}}{\sqrt{\rho_{0}}} \text { in } L^{2}, \quad \rho_{0}^{\delta} u_{0}^{\delta} \rightarrow m_{0} \text { in } L^{\frac{2 \gamma}{\gamma+1}}, \quad \rho_{0}^{\delta} v_{0}^{\delta} \rightarrow l_{0} \text { in } L^{\frac{2 \gamma}{\gamma+1}}
\end{gathered}
$$

as $\delta \rightarrow 0$. 
Let $\left(\rho_{\delta}, u_{\delta}, v_{\delta}, n_{\delta}\right)$ be a sequence of global weak solutions to

$$
\left\{\begin{array}{l}
\left(\rho_{\delta}\right)_{t}+\left(\rho_{\delta} u_{\delta}\right)_{x}=0, \quad \rho_{\delta}>0 \\
\left(\rho_{\delta} u \delta\right)_{t}+\left(\rho_{\delta} u_{\delta}^{2}\right)_{x}+\left(\rho_{\delta}^{\gamma}\right)_{x}=J_{\delta}^{1}-\left(n_{\delta}\right)_{x x}\left(n_{\delta}\right)_{x} \\
\left(\rho_{\delta} v_{\delta}\right)_{t}+\left(\rho_{\delta} u_{\delta} v_{\delta}\right)_{x}=J_{\delta}^{2} \\
\gamma_{1}\left(\dot{n}_{\delta}-\frac{1}{2}\left(v_{\delta}\right)_{x}\right)-\gamma_{2}\left(\left(u_{\delta}\right)_{x} \cos n_{\delta} \sin n_{\delta}+\frac{1}{2}\left(v_{\delta}\right)_{x}\left(1-2 \cos ^{2} n_{\delta}\right)\right)=\left(n_{\delta}\right)_{x x}
\end{array}\right.
$$

with the initial and boundary values

$$
\begin{gathered}
\left(\rho_{\delta}, u_{\delta}, v_{\delta}, n_{\delta}\right)(x, 0)=\left(\rho_{0}^{\delta}, u_{0}^{\delta}, v_{0}^{\delta}, n_{0}^{\delta}\right)(x) \\
u_{\delta}(0, t)=v_{\delta}(0, t)=u_{\delta}(1, t)=v_{\delta}(1, t)=0, \quad\left(n_{\delta}\right)_{x}(0, t)=\left(n_{\delta}\right)_{x}(1, t)=0 .
\end{gathered}
$$

Here $J_{\delta}^{1}$ and $J_{\delta}^{2}$ have the same forms as $J^{1}$ and $J^{2}$, but with $(u, v, n)$ replaced by $\left(u_{\delta}, v_{\delta}, n_{\delta}\right)$.

By Lemma 3.1-Lemma 3.3, we can find a subsequence $\left(\rho_{\delta}, u_{\delta}, v_{\delta}, n_{\delta}\right)$, still denoted as $\left(\rho_{\delta}, u_{\delta}, v_{\delta}, n_{\delta}\right)$, such that for any $T>0$, as $\delta \rightarrow 0$,

$$
\begin{gathered}
\rho_{\delta} \stackrel{*}{\rightarrow} \rho, \text { in } L^{\infty}\left(0, T ; L^{\gamma}\right), \quad \rho_{\delta} \rightarrow \rho, \text { in } L^{2 \gamma}([0,1] \times[0, T]), \\
\rho_{\delta}^{\gamma} \rightarrow \overline{\rho^{\gamma}}, \text { in } L^{2}([0,1] \times[0, T]), \\
u_{\delta} \rightarrow u, \text { in } L^{2}\left(0, T ; H_{0}^{1}\right), \quad v_{\delta} \rightarrow v, \text { in } L^{2}\left(0, T ; H_{0}^{1}\right), \\
n_{\delta} \stackrel{*}{\rightarrow} n, \text { in } L^{\infty}([0,1] \times[0, T]), \quad\left(n_{\delta}\right)_{x} \stackrel{*}{\rightarrow} n_{x}, \text { in } L^{\infty}\left(0, T ; L^{2}\right), \\
\left(n_{\delta}\right)_{t} \rightarrow n_{t}, \text { in } L^{2}([0,1] \times[0, T]), \quad\left(n_{\delta}\right)_{x x} \rightarrow n_{x x}, \text { in } L^{2}([0,1] \times[0, T]) .
\end{gathered}
$$

Since $\rho_{\delta}>0$, for any nonnegative function $f \in C_{0}^{\infty}((0,1) \times(0, T))$ it holds that

$$
\int_{0}^{T} \int_{0}^{1} \rho f=\lim _{\delta \rightarrow 0} \int_{0}^{T} \int_{0}^{1} \rho_{\delta} f \geq 0
$$

Since $f$ is arbitrary, we conclude that $\rho \geq 0$ a.e. in $(0,1) \times(0, T)$.

We need to show the limit $(\rho, u, v, n)$ is a solution to the system (4.1). We first state several compactness results that will be used in our proof.

Lemma 5.1 ([22]) Assume $X \subset E \subset Y$ are Banach spaces and $X \hookrightarrow \hookrightarrow E$ is compact. Then the following embeddings are compact

$$
\begin{gathered}
\left\{f: f \in L^{q}(0, T ; X), \frac{\partial f}{\partial t} \in L^{1}(0, T ; Y)\right\} \hookrightarrow \hookrightarrow L^{q}(0, T ; E), \text { for any } 1 \leq q \leq \infty, \\
\left\{f: f \in L^{\infty}(0, T ; X), \frac{\partial f}{\partial t} \in L^{r}(0, T ; Y)\right\} \hookrightarrow \hookrightarrow C([0, T] ; E), \text { for any } 1<r<\infty .
\end{gathered}
$$


Lemma 5.2 ([6]) Let $\bar{O} \subset \mathbb{R}^{n}$ be compact and $X$ be a separable Banach space. Assume that $f_{\delta}: \bar{O} \rightarrow X^{*}$ is a sequence of measurable functions such that for any $k$

$$
\operatorname{ess} \sup _{\bar{O}}\left\|f_{\delta}\right\|_{X^{*}} \leq N<\infty
$$

Moreover, the family of functions $\left\langle f_{\delta}, \Phi\right\rangle$ is equi-continuous for any $\Phi$ belonging to a dense subset of $X$. Then $f_{\delta} \in C(\bar{O} ; X-w)$ for any $k$, i.e., for any $g \in X *,\left\langle f_{\delta}, g\right\rangle \in C(\bar{O})$. Furthermore, there exists $f \in C(\bar{O} ; X-w)$ such that (after taking possible subsequences)

$$
f_{\delta} \rightarrow f, \quad \text { in } C(\bar{O} ; X-w)
$$

as $\delta \rightarrow 0$.

First observe that $\rho_{\delta} \in L^{2 \gamma}([0,1] \times[0, T])$ and $u_{\delta} \in L^{2}\left(0, T ; H_{0}^{1}\right) \subset L^{2}\left(0, T ; L^{\infty}\right)$ imply

$$
\rho_{\delta} u_{\delta} \in L^{\frac{2 \gamma}{\gamma+1}}\left(0, T ; L^{2 \gamma}\right), \quad\left(\rho_{\delta}\right)_{t}=-\left(\rho_{\delta} u_{\delta}\right)_{x} \in L^{\frac{2 \gamma}{\gamma+1}}\left(0, T ; H^{-1}\right) .
$$

By Lemma 5.1 and Lemma 5.2, and $\frac{2 \gamma}{\gamma+1}>1, \rho_{\delta} \in L^{\infty}\left(0, T ; L^{\gamma}\right), L^{\gamma} \hookrightarrow \hookrightarrow H^{-1}$, we conclude

$$
\rho_{\delta} \rightarrow \rho, \text { in } C\left(0, T ; L^{\gamma}-\omega\right), \quad \rho_{\delta} \rightarrow \rho, \text { in } C\left(0, T ; H^{-1}\right),
$$

where $f \in C(0, T ; X-\omega)$ if for any $g \in X^{*},\langle f(t), g\rangle \in C([0, T])$. Hence

$$
\rho_{\delta} u_{\delta} \rightarrow \rho u, \text { in } \mathcal{D}^{\prime}((0,1) \times(0, T)), \quad \rho_{\delta} v_{\delta} \rightarrow \rho v, \text { in } \mathcal{D}^{\prime}((0,1) \times(0, T)),
$$

and furthermore

$$
\rho_{t}+(\rho u)_{x}=0, \text { in } \mathcal{D}^{\prime}((0,1) \times(0, T)) .
$$

By (5.11), it also holds that

$$
\rho(x, 0)=\rho_{0}(x) \text {, weakly in } L^{\gamma}([0,1]) .
$$

By the fact $\left(n_{\delta}\right)_{t} \in L^{2}\left(0, T ; L^{2}\right)$, (5.9) and (5.10), we can apply Lemma 5.1 to obtain

$$
n_{\delta} \rightarrow n, \text { in } C([0,1] \times[0, T]), \quad n_{\delta} \rightarrow n, \text { in } L^{2}\left(0, T ; C^{1}\right),
$$

Combining with (5.8)-(5.10), we can show the limit $n$ satisfies the following equation:

$$
\gamma_{1}\left(\dot{n}-\frac{1}{2} v_{x}\right)-\gamma_{2}\left(u_{x} \cos n \sin n+\frac{1}{2} v_{x}\left(1-2 \cos ^{2} n\right)\right)=n_{x x} .
$$

By (5.15), it also holds that

$$
n(x, 0)=n_{0}(x), \text { in }[0,1] .
$$

By the fact $\sqrt{\rho_{\delta}} \in L^{2 \gamma}([0,1] \times[0, T])$ and $\sqrt{\rho_{\delta}} u_{\delta} \in L^{\infty}\left(0, T ; L^{2}\right)$, it holds

$$
\rho_{\delta} u_{\delta} \in L^{\infty}\left(0, T ; L^{\frac{2 \gamma}{\gamma+1}}\right) .
$$


Combining with (5.8), we have

$$
\rho_{\delta} u_{\delta}^{2} \rightarrow \rho u^{2}, \text { in } L^{2}\left(0, T ; L^{\frac{2 \gamma}{\gamma+1}}\right)
$$

By the second equation of system (5.3), we have

$$
\left(\rho_{\delta} u_{\delta}\right)_{t}=-\left(\rho_{\delta} u_{\delta}^{2}\right)_{x}-\left(\rho_{\delta}^{\gamma}\right)_{x}+J_{\delta}^{1}-\left(n_{\delta}\right)_{x x}\left(n_{\delta}\right)_{x} \in L^{2}\left(0, T ; W^{-1, \frac{2 \gamma}{\gamma+1}}\right),
$$

where $J_{\delta}^{1}$ has the same form as $J^{1}$, but with $(u, v, n)$ replaced by $\left(u_{\delta}, v_{\delta}, n_{\delta}\right)$. By using Lemma 5.1 and Lemma 5.2, we conclude

$$
\rho_{\delta} u_{\delta} \rightarrow \rho u, \text { in } C\left(0, T ; L^{\frac{2 \gamma}{\gamma+1}}-\omega\right), \quad \rho_{\delta} u_{\delta} \rightarrow \rho u, \text { in } C\left(0, T ; H^{-1}\right) .
$$

Combining with (5.8), we conclude that

$$
\rho_{\delta} u_{\delta}^{2} \rightarrow \rho u^{2}, \text { in } \mathcal{D}^{\prime}((0,1) \times(0, T)) .
$$

Therefore

$$
(\rho u)_{t}+\left(\rho u^{2}\right)_{x}+\left(\overline{\rho^{\gamma}}\right)_{x}=J^{1}-n_{x x} n_{x}, \text { in } \mathcal{D}^{\prime}((0,1) \times(0, T)) .
$$

By (5.19), it holds that

$$
\rho u(x, 0)=m_{0}(x), \text { weakly in } L^{\frac{2 \gamma}{\gamma+1}}([0,1]) .
$$

Similarly, we can also prove that

$$
\begin{gathered}
(\rho v)_{t}+(\rho u v)_{x}=J^{2}, \text { in } \mathcal{D}^{\prime}((0,1) \times(0, T)), \\
\rho v(x, 0)=n_{0}(x), \text { weakly in } L^{\frac{2 \gamma}{\gamma+1}}([0,1]) .
\end{gathered}
$$

By (5.20), for some $t \in(0, T)$ and small $\epsilon>0$, it holds

$$
\frac{1}{\epsilon} \int_{t}^{t+\epsilon} \int_{0}^{1} \rho u^{2}=\frac{1}{\epsilon} \int_{t}^{t+\epsilon} \lim _{\delta \rightarrow 0} \int_{0}^{1} \rho_{\delta} u_{\delta}^{2} \leq \frac{1}{\epsilon} \int_{t}^{t+\epsilon} \varlimsup_{\delta \rightarrow 0} \int_{0}^{1} \rho_{\delta} u_{\delta}^{2} .
$$

Sending $\epsilon \rightarrow 0^{+}$and using the Lebesgue Differentiation Theorem, we obtain

$$
\int_{0}^{1} \rho u^{2} \leq \varlimsup_{\delta \rightarrow 0} \int_{0}^{1} \rho_{\delta} u_{\delta}^{2}
$$

for a.e. $t \in(0, T)$. Combining this limit with the lower semicontinuity, we can prove the energy inequality is valid.

The only thing left is to show $\overline{\rho^{\gamma}}=\rho^{\gamma}$. To this end, we denote

$$
A(n)=\left(A_{i j}(n)\right)_{2 \times 2}
$$

where the elements of $A_{i j}$ are given as follows

$$
A_{11}(n)=\left(\alpha_{0}+\alpha_{5}+\alpha_{6}+\alpha_{8}\right) \cos ^{2} n+\alpha_{1} \cos ^{4} n+\left(\alpha_{4}+\alpha_{7}\right)
$$




$$
\begin{gathered}
A_{12}(n)=\alpha_{0} \cos n \sin n+\alpha_{1} \cos ^{3} n \sin n+\frac{1}{2}\left(\alpha_{2}+\alpha_{3}+\alpha_{5}+\alpha_{6}\right) \cos n \sin n \\
A_{21}(n)=\alpha_{1} \cos ^{3} n \sin n+\left(\alpha_{6}+\alpha_{8}\right) \cos n \sin n \\
A_{22}(n)=\alpha_{1} \cos ^{2} n \sin ^{2} n+\frac{1}{2}\left(-\alpha_{2}+\alpha_{5}\right) \cos ^{2} n+\frac{1}{2}\left(\alpha_{3}+\alpha_{6}\right) \sin ^{2} n+\frac{1}{2} \alpha_{4} .
\end{gathered}
$$

By the relations (1.6), direct computations imply that there exist two positive constants $\lambda, \Lambda<\infty$ such that for any $\mathbf{y} \in \mathbb{R}^{2}$

$$
\lambda|\mathbf{y}|^{2} \leq \mathbf{y}^{T} A(n) \mathbf{y} \leq \Lambda|\mathbf{y}|^{2} .
$$

In fact

$$
\begin{aligned}
\mathbf{y}^{T} A(n) \mathbf{y}= & A_{11}(n) y_{1}^{2}+\left(A_{12}(n)+A_{21}(n)\right) y_{1} y_{2}+A_{22}(n) y_{2}^{2} \\
= & {\left[\left(\alpha_{0}+\alpha_{5}+\alpha_{6}+\alpha_{8}\right) \cos ^{2} n+\alpha_{1} \cos ^{4} n+\left(\alpha_{4}+\alpha_{7}\right)\right] y_{1}^{2} } \\
& +\left[\left(\alpha_{0}+\alpha_{6}+\alpha_{8}\right) \cos n \sin n+2 \alpha_{1} \cos ^{3} n \sin n+\frac{1}{2}\left(\alpha_{2}+\alpha_{3}+\alpha_{5}+\alpha_{6}\right) \cos n \sin n\right] y_{1} y_{2} \\
& +\left[\alpha_{1} \cos ^{2} n \sin ^{2} n+\frac{1}{2}\left(-\alpha_{2}+\alpha_{5}\right) \cos ^{2} n+\frac{1}{2}\left(\alpha_{3}+\alpha_{6}\right) \sin ^{2} n+\frac{1}{2} \alpha_{4}\right] y_{2}^{2} \\
= & \frac{1}{4}\left(\frac{\gamma_{2}}{\sqrt{\gamma_{1}}} y_{1} \sin (2 n)+\frac{1}{\sqrt{\gamma_{1}}}\left(\gamma_{1}-\gamma_{2} \cos (2 n)\right) y_{2}\right)^{2} \\
& +\frac{1}{4}\left(-\alpha_{1}-\frac{\gamma_{2}^{2}}{\gamma_{1}}\right) y_{1}^{2}+\left(\alpha_{4}+\alpha_{7}\right) y_{1}^{2}+\frac{1}{4}\left(2 \alpha_{4}+\alpha_{5}+\alpha_{6}-\frac{\gamma_{2}^{2}}{\gamma_{1}}\right) y_{2}^{2} \\
& \frac{1}{4}\left(\alpha_{1}+\frac{\gamma_{2}^{2}}{\gamma_{1}}\right)\left(y_{1} \cos (2 n)+y_{2} \sin (2 n)\right)^{2} \\
& +\left(\alpha_{0}+\alpha_{1}+\alpha_{5}+\alpha_{6}+\alpha_{8}\right)\left[\left(y_{1} \cos n+\frac{1}{2} y_{2} \sin n\right)^{2}-\frac{1}{4} y_{2}^{2} \sin ^{2} n\right] .
\end{aligned}
$$

Therefore

$$
\begin{aligned}
\mathbf{y}^{T} A(n) \mathbf{y} \geq & \frac{1}{4}\left(-\alpha_{1}-\frac{\gamma_{2}^{2}}{\gamma_{1}}\right) y_{1}^{2}+\left(\alpha_{4}+\alpha_{7}\right) y_{1}^{2}+\frac{1}{4}\left(2 \alpha_{4}+\alpha_{5}+\alpha_{6}-\frac{\gamma_{2}^{2}}{\gamma_{1}}\right) y_{2}^{2} \\
& -\frac{1}{4}\left(\alpha_{0}+\alpha_{1}+\alpha_{5}+\alpha_{6}+\alpha_{8}\right) y_{2}^{2} \sin ^{2} n
\end{aligned}
$$

If we take

$$
\lambda=\min \left\{\left(\alpha_{4}+\alpha_{7}\right)-\frac{1}{4}\left(\alpha_{1}+\frac{\gamma_{2}^{2}}{\gamma_{1}}\right),\left(2 \alpha_{4}+\alpha_{5}+\alpha_{6}-\frac{\gamma_{2}^{2}}{\gamma_{1}}\right)-\left(\alpha_{0}+\alpha_{1}+\alpha_{5}+\alpha_{6}+\alpha_{8}\right)\right\},
$$

then by the relation (1.6), we know that $\lambda>0$ and we have shown the estimate (5.25).

By the definition of $A(n)$, we see that the matrix valued function $A(\cdot) \in C^{\infty}$. By the estimate (5.25), the inverse matrix function $A^{-1}$ exists and

$$
\frac{d}{d n}\left(A^{-1}(n)\right)=A^{-1} \frac{d}{d n}(A(n)) A^{-1}
$$

The equations for $\mathbf{u}=(u, v)^{T}$ can be written as

$$
\rho \mathbf{u}_{t}+\rho u \mathbf{u}_{x}+\mathbf{P}_{x}=\left(A(n) \mathbf{u}_{x}\right)_{x}+\left(B_{1}(n)\right)_{x}-B_{2}(n)
$$


where

$$
\begin{gathered}
\mathbf{P}=\left(\overline{\rho^{\gamma}}, 0\right)^{T}, \\
B_{1}(n)=\left(\left(\alpha_{2}+\alpha_{3}\right) \dot{n} \cos n \sin n, \alpha_{2} \dot{n} \cos ^{2} n-\alpha_{3} \dot{n} \sin ^{2} n\right)^{T}, \\
B_{2}(n)=\left(n_{x x} n_{x}, 0\right)^{T} .
\end{gathered}
$$

Similarly, we can rewrite the equations for $\mathbf{u}_{\delta}=\left(u_{\delta}, v_{\delta}\right)^{T}, \mathbf{P}_{\delta}=\left(\rho_{\delta}^{\gamma}, 0\right)^{T}$ in the similar form

$$
\rho_{\delta}\left(\mathbf{u}_{\delta}\right)_{t}+\rho_{\delta} u_{\delta}\left(\mathbf{u}_{\delta}\right)_{x}+\left(\mathbf{P}_{\delta}\right)_{x}=\left(A\left(n_{\delta}\right)\left(\mathbf{u}_{\delta}\right)_{x}\right)_{x}+\left(B_{1}\left(n_{\delta}\right)\right)_{x}-B_{2}\left(n_{\delta}\right) .
$$

Denote

$$
\mathcal{H}=\mathbf{u}_{x}-A^{-1}(n) \mathbf{P}, \quad \mathcal{H}_{\delta}=\left(\mathbf{u}_{\delta}\right)_{x}-A^{-1}\left(n_{\delta}\right) \mathbf{P}_{\delta} .
$$

We have the following lemma.

Lemma 5.3 As $\delta \rightarrow 0$, it holds

$$
\rho_{\delta} \mathcal{H}_{\delta} \rightarrow \rho \mathcal{H}, \text { in } \mathcal{D}^{\prime}((0,1) \times(0, T)) \text {. }
$$

Proof. The main difficulty of the proof arises from $\rho u \notin L^{2}$. To overcome it, we need to mollify the density $\rho$ by $\langle\hat{\rho}\rangle_{\sigma}=\eta_{\sigma} * \hat{\rho}$, where $\eta_{\sigma}=\frac{1}{\sigma} \sigma(\dot{\bar{\sigma}})$ is the standard mollifier, $\hat{f}$ is the zero extension of $f$ from $(0,1)$ to $\mathbb{R}$. By Lemma 3.3 in [6], the zero-extension of $\hat{\rho}$ still satisfies the same equation

$$
(\hat{\rho})_{t}+(\hat{\rho} \hat{u})_{x}=0, \quad \text { in } \mathcal{D}^{\prime}(\mathbb{R} \times(0, T)) .
$$

Denote $\tau^{\sigma}=\left(\langle\hat{\rho}\rangle_{\sigma} \hat{u}\right)_{x}-\left\langle(\hat{\rho} \hat{u})_{x}\right\rangle_{\sigma}$. By Lemma 2.3 in [18], we know that $\tau^{\sigma} \in L^{\frac{2 \gamma}{\gamma+1}}(\mathbb{R} \times(0, T)$, and as $\sigma \rightarrow 0$

$$
\tau^{\sigma} \rightarrow 0, \quad \text { in } L^{1}(\mathbb{R} \times(0, T)) .
$$

Taking the standard mollifier as the test function, we obtain

$$
\left(\langle\hat{\rho}\rangle_{\sigma}\right)_{t}+\left(\langle\hat{\rho}\rangle_{\sigma} \hat{u}\right)_{x}=\tau^{\sigma}, \quad \text { in } \mathcal{D}^{\prime}(\mathbb{R} \times(0, T))
$$

Similarly, it also hold for the approximate solutions

$$
\left(\left\langle\hat{\rho}_{\delta}\right\rangle_{\sigma}\right)_{t}+\left(\left\langle\hat{\rho}_{\delta}\right\rangle_{\sigma} \hat{u}_{\delta}\right)_{x}=\tau_{\delta}^{\sigma}, \quad \text { in } \mathcal{D}^{\prime}(\mathbb{R} \times(0, T)),
$$

where $\tau_{\delta}^{\sigma}$ has the same form as $\tau^{\sigma}$, but with $\rho, u$ replaced by $\rho_{\delta}, u_{\delta}$. We also know that, for any $\delta>0, \tau_{\delta}^{\sigma} \in L^{\frac{2 \gamma}{\gamma+1}}(\mathbb{R} \times(0, T)$, and as $\sigma \rightarrow 0$

$$
\tau_{\delta}^{\sigma} \rightarrow 0, \quad \text { in } L^{1}(\mathbb{R} \times(0, T))
$$


Multiplying the equation (5.27) by $\varphi \phi A^{-1}\left(n_{\delta}\right) \int_{0}^{x}\left\langle\hat{\rho}_{\delta}\right\rangle_{\sigma}$ from left for any $\varphi \in C_{0}^{\infty}(0, T)$ and $\phi \in C_{0}^{\infty}(0,1)$, and integrating by parts, we obtain

$$
\begin{aligned}
& \int_{0}^{T} \int_{0}^{1} \varphi \phi \mathcal{H}_{\delta}\left\langle\hat{\rho}_{\delta}\right\rangle_{\sigma} \\
= & \int_{0}^{T} \int_{0}^{1} \varphi^{\prime} \phi \rho_{\delta} A^{-1}\left(n_{\delta}\right) \mathbf{u}_{\delta} \int_{0}^{x}\left\langle\hat{\rho}_{\delta}\right\rangle_{\sigma}+\int_{0}^{T} \int_{0}^{1} \varphi \phi \rho_{\delta} A^{-1}\left(n_{\delta}\right) \mathbf{u}_{\delta}\left(\int_{0}^{x}\left\langle\hat{\rho}_{\delta}\right\rangle_{\sigma}\right)_{t} \\
& +\int_{0}^{T} \int_{0}^{1} \varphi \phi \rho_{\delta}\left(A^{-1}\left(n_{\delta}\right)\right)_{t} \mathbf{u}_{\delta} \int_{0}^{x}\left\langle\hat{\rho}_{\delta}\right\rangle_{\sigma}+\int_{0}^{T} \int_{0}^{1} \varphi \phi^{\prime} \rho_{\delta} u_{\delta} A^{-1}\left(n_{\delta}\right) \mathbf{u}_{\delta} \int_{0}^{x}\left\langle\hat{\rho}_{\delta}\right\rangle_{\sigma} \\
& +\int_{0}^{T} \int_{0}^{1} \varphi \phi \rho_{\delta}\left\langle\hat{\rho}_{\delta}\right\rangle_{\sigma} u_{\delta} A^{-1}\left(n_{\delta}\right) \mathbf{u}_{\delta}+\int_{0}^{T} \int_{0}^{1} \varphi \phi \rho_{\delta} u_{\delta}\left(A^{-1}\left(n_{\delta}\right)\right)_{x} \mathbf{u}_{\delta} \int_{0}^{x}\left\langle\hat{\rho}_{\delta}\right\rangle_{\sigma} \\
& +\int_{0}^{T} \int_{0}^{1} \varphi \phi A^{-1}\left(n_{\delta}\right)\left(B_{1}\left(n_{\delta}\right)\right)_{x} \int_{0}^{x}\left\langle\hat{\rho}_{\delta}\right\rangle_{\sigma}-\int_{0}^{T} \int_{0}^{1} \varphi \phi A^{-1}\left(n_{\delta}\right) B_{2}\left(n_{\delta}\right) \int_{0}^{x}\left\langle\hat{\rho}_{\delta}\right\rangle_{\sigma} \\
& -\int_{0}^{T} \int_{0}^{1} \varphi \phi^{\prime} \mathcal{H}_{\delta} \int_{0}^{x}\left\langle\hat{\rho}_{\delta}\right\rangle_{\sigma}-\int_{0}^{T} \int_{0}^{1} \varphi \phi A^{-1}\left(n_{\delta}\right)\left(A\left(n_{\delta}\right)\right)_{x} \mathcal{H}_{\delta} \int_{0}^{x}\left\langle\hat{\rho}_{\delta}\right\rangle_{\sigma} .
\end{aligned}
$$

The equation (5.32) implies

$$
\frac{\partial}{\partial t}\left(\int_{0}^{x}\left\langle\hat{\rho}_{\delta}\right\rangle_{\sigma}\right)=-\left\langle\hat{\rho}_{\delta}\right\rangle_{\sigma} \hat{u}_{\delta}+\tau_{\delta}^{\sigma}
$$

Using this fact, we have

$$
\begin{aligned}
& \int_{0}^{T} \int_{0}^{1} \varphi \phi \mathcal{H}_{\delta}\left\langle\hat{\rho}_{\delta}\right\rangle_{\sigma} \\
= & \int_{0}^{T} \int_{0}^{1} \varphi^{\prime} \phi \rho_{\delta} A^{-1}\left(n_{\delta}\right) \mathbf{u}_{\delta} \int_{0}^{x}\left\langle\hat{\rho}_{\delta}\right\rangle_{\sigma}+\int_{0}^{T} \int_{0}^{1} \varphi \phi \rho_{\delta} A^{-1}\left(n_{\delta}\right) \mathbf{u}_{\delta} \int_{0}^{x} \tau_{\delta}^{\sigma} \\
& +\int_{0}^{T} \int_{0}^{1} \varphi \phi \rho_{\delta}\left(A^{-1}\left(n_{\delta}\right)\right)_{t} \mathbf{u}_{\delta} \int_{0}^{x}\left\langle\hat{\rho}_{\delta}\right\rangle_{\sigma}+\int_{0}^{T} \int_{0}^{1} \varphi \phi^{\prime} \rho_{\delta} u_{\delta} A^{-1}\left(n_{\delta}\right) \mathbf{u}_{\delta} \int_{0}^{x}\left\langle\hat{\rho}_{\delta}\right\rangle_{\sigma} \\
& +\int_{0}^{T} \int_{0}^{1} \varphi \phi \rho_{\delta} u_{\delta}\left(A^{-1}\left(n_{\delta}\right)\right)_{x} \mathbf{u}_{\delta} \int_{0}^{x}\left\langle\hat{\rho}_{\delta}\right\rangle_{\sigma} \\
& +\int_{0}^{T} \int_{0}^{1} \varphi \phi A^{-1}\left(n_{\delta}\right)\left(B_{1}\left(n_{\delta}\right)\right)_{x} \int_{0}^{x}\left\langle\hat{\rho}_{\delta}\right\rangle_{\sigma}-\int_{0}^{T} \int_{0}^{1} \varphi \phi A^{-1}\left(n_{\delta}\right) B_{2}\left(n_{\delta}\right) \int_{0}^{x}\left\langle\hat{\rho}_{\delta}\right\rangle_{\sigma} \\
& -\int_{0}^{T} \int_{0}^{1} \varphi \phi^{\prime} \mathcal{H}_{\delta} \int_{0}^{x}\left\langle\hat{\rho}_{\delta}\right\rangle_{\sigma}-\int_{0}^{T} \int_{0}^{1} \varphi \phi A^{-1}\left(n_{\delta}\right)\left(A\left(n_{\delta}\right)\right)_{x} \mathcal{H}_{\delta} \int_{0}^{x}\left\langle\hat{\rho}_{\delta}\right\rangle_{\sigma} .
\end{aligned}
$$

By the Lebesgue Dominated Convergence theorem and (5.33), we may take the limit $\sigma \rightarrow 0$ and 
get

$$
\begin{aligned}
& \int_{0}^{T} \int_{0}^{1} \varphi \phi \mathcal{H}_{\delta} \rho_{\delta} \\
= & \int_{0}^{T} \int_{0}^{1} \varphi^{\prime} \phi \rho_{\delta} A^{-1}\left(n_{\delta}\right) \mathbf{u}_{\delta} \int_{0}^{x} \rho_{\delta}+\int_{0}^{T} \int_{0}^{1} \varphi \phi \rho_{\delta}\left(A^{-1}\left(n_{\delta}\right)\right)_{t} \mathbf{u}_{\delta} \int_{0}^{x} \rho_{\delta} \\
& +\int_{0}^{T} \int_{0}^{1} \varphi \phi^{\prime} \rho_{\delta} u_{\delta} A^{-1}\left(n_{\delta}\right) \mathbf{u}_{\delta} \int_{0}^{x} \rho_{\delta}+\int_{0}^{T} \int_{0}^{1} \varphi \phi \rho_{\delta} u_{\delta}\left(A^{-1}\left(n_{\delta}\right)\right)_{x} \mathbf{u}_{\delta} \int_{0}^{x} \rho_{\delta} \\
& +\int_{0}^{T} \int_{0}^{1} \varphi \phi A^{-1}\left(n_{\delta}\right)\left(B_{1}\left(n_{\delta}\right)\right)_{x} \int_{0}^{x} \rho_{\delta}-\int_{0}^{T} \int_{0}^{1} \varphi \phi A^{-1}\left(n_{\delta}\right) B_{2}\left(n_{\delta}\right) \int_{0}^{x} \rho_{\delta} \\
& -\int_{0}^{T} \int_{0}^{1} \varphi \phi^{\prime} \mathcal{H}_{\delta} \int_{0}^{x} \rho_{\delta}-\int_{0}^{T} \int_{0}^{1} \varphi \phi A^{-1}\left(n_{\delta}\right)\left(A\left(n_{\delta}\right)\right)_{x} \mathcal{H}_{\delta} \int_{0}^{x} \rho_{\delta} .
\end{aligned}
$$

By the definition of $B_{2}\left(n_{\delta}\right)$ and integration by parts, we obtain

$$
\begin{aligned}
& -\int_{0}^{T} \int_{0}^{1} \varphi \phi A^{-1}\left(n_{\delta}\right) B_{2}\left(n_{\delta}\right) \int_{0}^{x} \rho_{\delta} \\
= & \frac{1}{2} \int_{0}^{T} \int_{0}^{1} \varphi \phi^{\prime} A^{-1}\left(n_{\delta}\right)\left(\left|\left(n_{\delta}\right)_{x}\right|^{2}, 0\right)^{T} \int_{0}^{x} \rho_{\delta}+\frac{1}{2} \int_{0}^{T} \int_{0}^{1} \varphi \phi\left(A^{-1}\left(n_{\delta}\right)\right)_{x}\left(\left|\left(n_{\delta}\right)_{x}\right|^{2}, 0\right)^{T} \int_{0}^{x} \rho_{\delta} \\
& +\frac{1}{2} \int_{0}^{T} \int_{0}^{1} \varphi \phi \rho_{\delta} A^{-1}\left(n_{\delta}\right)\left(\left|\left(n_{\delta}\right)_{x}\right|^{2}, 0\right)^{T} .
\end{aligned}
$$

By the definition of $B_{1}\left(n_{\delta}\right)$, we obtain

$$
\begin{aligned}
& \int_{0}^{T} \int_{0}^{1} \varphi \phi A^{-1}\left(n_{\delta}\right)\left(B_{1}\left(n_{\delta}\right)\right)_{x} \int_{0}^{x} \rho_{\delta} \\
= & \int_{0}^{T} \int_{0}^{1} \varphi \phi\left(A^{-1}\left(n_{\delta}\right) B_{1}\left(n_{\delta}\right)\right)_{x} \int_{0}^{x} \rho_{\delta}-\int_{0}^{T} \int_{0}^{1} \varphi \phi\left(A^{-1}\left(n_{\delta}\right)\right)_{x} B_{1}\left(n_{\delta}\right) \int_{0}^{x} \rho_{\delta} .
\end{aligned}
$$

It is not hard to see that there is a vector function $\mathcal{F}\left(n_{\delta}\right)$ (smooth in $n_{\delta}$ ) such that

$$
A^{-1}\left(n_{\delta}\right) B_{1}\left(n_{\delta}\right)=\mathcal{F}_{t}\left(n_{\delta}\right)+u_{\delta} \mathcal{F}_{x}\left(n_{\delta}\right)
$$

Then

$$
\begin{aligned}
& \int_{0}^{T} \int_{0}^{1} \varphi \phi A^{-1}\left(n_{\delta}\right)\left(B_{1}\left(n_{\delta}\right)\right)_{x} \int_{0}^{x} \rho_{\delta} \\
= & -\int_{0}^{T} \int_{0}^{1} \varphi^{\prime} \phi \mathcal{F}_{x}\left(n_{\delta}\right) \int_{0}^{x} \rho_{\delta}-\int_{0}^{T} \int_{0}^{1} \varphi \phi^{\prime} u_{\delta} \mathcal{F}_{x}\left(n_{\delta}\right) \int_{0}^{x} \rho_{\delta} \\
& -\int_{0}^{T} \int_{0}^{1} \varphi \phi\left(A^{-1}\left(n_{\delta}\right)\right)_{x} B_{1}\left(n_{\delta}\right) \int_{0}^{x} \rho_{\delta} .
\end{aligned}
$$

To estimate the second term on right side of (15.34), we use $\varphi \phi n$ as the test function for the first equation of (5.3) to obtain

$$
\int_{0}^{T} \int_{0}^{1} \varphi \phi \rho_{\delta}\left(n_{\delta}\right)_{t}=-\int_{0}^{T} \int_{0}^{1} \varphi^{\prime} \phi \rho_{\delta} n_{\delta}-\int_{0}^{T} \int_{0}^{1} \varphi \rho_{\delta} u_{\delta}\left(n_{\delta} \phi\right)_{x} .
$$


Similarly, it holds

$$
\int_{0}^{T} \int_{0}^{1} \varphi \phi \rho n_{t}=-\int_{0}^{T} \int_{0}^{1} \varphi^{\prime} \phi \rho n-\int_{0}^{T} \int_{0}^{1} \varphi \rho u(n \phi)_{x}
$$

Taking the difference, and using (5.6), (5.12) and (5.15), we have

$$
\rho_{\delta}\left(n_{\delta}\right)_{t} \rightarrow \rho n_{t}, \text { in } \mathcal{D}^{\prime}((0,1) \times(0, T)) .
$$

Furthermore, since

$$
\int_{0}^{x} \rho_{\delta} \in L^{\infty}\left(0, T ; W^{1, \gamma}\right), \quad \frac{\partial}{\partial t}\left(\int_{0}^{x} \rho_{\delta}\right)=-\rho_{\delta} u_{\delta} \in L^{\infty}\left(0, T ; L^{\frac{2 \gamma}{\gamma+1}}\right)
$$

we obtain by Lemma 5.1 and $(5.6)$

$$
\int_{0}^{x} \rho_{\delta} \rightarrow \int_{0}^{x} \rho, \quad \text { in } C([0,1] \times[0, T]), \quad \text { as } \delta \rightarrow 0 .
$$

Now, we are ready to take limit in (5.34). Letting $\delta \rightarrow 0$ in (5.34) (5.35) and (5.36), and using the facts (5.38), (5.37), (5.6)

$$
\begin{aligned}
& \lim _{\delta \rightarrow 0} \int_{0}^{T} \int_{0}^{1} \varphi \phi \mathcal{H}_{\delta} \rho_{\delta} \\
= & \int_{0}^{T} \int_{0}^{1} \varphi^{\prime} \phi \rho A^{-1}(n) \mathbf{u} \int_{0}^{x} \rho+\int_{0}^{T} \int_{0}^{1} \varphi \phi \rho\left(A^{-1}(n)\right)_{t} \mathbf{u} \int_{0}^{x} \rho \\
& +\int_{0}^{T} \int_{0}^{1} \varphi \phi^{\prime} \rho u A^{-1}(n) \mathbf{u} \int_{0}^{x} \rho+\int_{0}^{T} \int_{0}^{1} \varphi \phi \rho u\left(A^{-1}(n)\right)_{x} \mathbf{u} \int_{0}^{x} \rho \\
& +\int_{0}^{T} \int_{0}^{1} \varphi \phi A^{-1}(n)\left(B_{1}(n)\right)_{x} \int_{0}^{x} \rho-\int_{0}^{T} \int_{0}^{1} \varphi \phi A^{-1}(n) B_{2}(n) \int_{0}^{x} \rho \\
& -\int_{0}^{T} \int_{0}^{1} \varphi \phi^{\prime} \mathcal{H} \int_{0}^{x} \rho-\int_{0}^{T} \int_{0}^{1} \varphi \phi A(n)\left(A^{-1}(n)\right)_{x} \mathcal{H} \int_{0}^{x} \rho .
\end{aligned}
$$

We may go through the same arguments for $\rho$ and $u$, and show that right side of (5.39) is exactly

$$
\int_{0}^{T} \int_{0}^{1} \varphi \phi \mathcal{H} \rho
$$

which completes the proof of the lemma.

We also need the following result.

Lemma 5.4 ([6]) Let $\bar{O} \subset \mathbb{R}^{n}$ be a measurable set and $f_{k} \in L^{1}\left(O ; \mathbb{R}^{N}\right)$ for $k \in \mathbb{Z}_{+}$such that

$$
f_{k} \rightarrow f, \quad \text { in } L^{1}\left(O ; \mathbb{R}^{N}\right)
$$

Let $\Phi: \mathbb{R}^{N} \rightarrow(-\infty, \infty]$ be a lower semi-continuous convex function such that $\Phi\left(f_{k}\right) \in L^{1}(O)$ for any $k$ and

$$
\Phi\left(f_{k}\right) \rightarrow \overline{\Phi(f)}, \quad \text { in } L^{1}(O)
$$


Then

$$
\Phi(f) \leq \overline{\Phi(f)}, \quad \text { a.e. in } O .
$$

Moreover, if $\Phi$ is strictly convex on an open convex set $U \subset \mathbb{R}^{N}$ and

$$
\Phi(f)=\overline{\Phi(f)}, \quad \text { a.e. in } O
$$

then

$$
f_{k} \rightarrow f, \quad \text { for a.e. } y \in\{y \in O \mid f(y) \in U\}
$$

The proof of Theorem 1.2 will be completed by the following Lemma.

Lemma 5.5 As $\delta \rightarrow 0$, it holds

$$
\lim _{\delta \rightarrow 0} \int_{0}^{T} \int_{0}^{1} \rho_{\delta} \log \left(\rho_{\delta}\right)=\int_{0}^{T} \int_{0}^{1} \rho \log \rho .
$$

Proof. By Proposition 4.2 in [7], if $\rho \in L^{2}((0,1) \times(0, T)), u \in L^{2}\left(0, T ; H_{0}^{1}\right)$ solves the equation

$$
\rho_{t}+(\rho u)_{x}=0, \quad \text { in } \mathcal{D}^{\prime}((0,1) \times(0, T))
$$

then

$$
(b(\rho))_{t}+(b(\rho) u)_{x}+\left(b^{\prime}(\rho) \rho-b(\rho)\right) u_{x}=0, \quad \text { in } \mathcal{D}^{\prime}((0,1) \times(0, T))
$$

for any $b \in C^{1}(\mathbb{R})$ such that $b^{\prime}(x) \equiv 0$ for all large enough $x \in \mathbb{R}$.

For any positive integers $j, K$, we may take a family of functions $b_{K}^{j} \in C^{1}(\mathbb{R})$ with

$$
b_{K}^{j}(x)= \begin{cases}\left(x+\frac{1}{j}\right) \log \left(x+\frac{1}{j}\right), & \text { if } 0 \leq x \leq K, \\ \left(K+1+\frac{1}{j}\right) \log \left(K+1+\frac{1}{j}\right), & \text { if } x \geq K+1 .\end{cases}
$$

Since $\rho \in L^{\infty}\left(0, T ; L^{\gamma}\right)$, we have $\rho<\infty$ a.e. in $(0,1) \times(0, T)$. This implies that $b_{K}^{j}(\rho) \rightarrow$ $\left(\rho+\frac{1}{j}\right) \log \left(\rho+\frac{1}{j}\right)$ a.e. in $(0,1) \times(0, T)$ as $K \rightarrow \infty$. Hence, by using the Lebesgue Dominated Convergence theorem, we conclude

$$
\left(\left(\rho+\frac{1}{j}\right) \log \left(\rho+\frac{1}{j}\right)\right)_{t}+\left(\left(\rho+\frac{1}{j}\right) \log \left(\rho+\frac{1}{j}\right) u\right)_{x}+\left(\rho-\frac{1}{j} \log \left(\rho+\frac{1}{j}\right)\right) u_{x}=0,
$$

in $\mathcal{D}^{\prime}((0,1) \times(0, T))$.

It is easy to see that $\left(\rho+\frac{1}{j}\right) \log \left(\rho+\frac{1}{j}\right) \in L^{2}((0,1) \times(0, T))$ since $\rho \in L^{2 \gamma}((0,1) \times(0, T))$. By Lemma 3.3 in [6], the zero-extension of $\rho$ outside $(0,1)$ satisfies the same equation. By the mollification, the integration by parts and the limiting process, we may take the test function to be the constant 1 so that

$$
\begin{aligned}
& \int_{0}^{T} \int_{0}^{1} \rho u_{x} \\
= & \int_{0}^{1}\left(\rho_{0}+\frac{1}{j}\right) \log \left(\rho_{0}+\frac{1}{j}\right)-\int_{0}^{1}\left(\rho+\frac{1}{j}\right) \log \left(\rho+\frac{1}{j}\right)(T) \\
& +\frac{1}{j} \int_{0}^{T} \int_{0}^{1} u_{x} \log \left(\rho+\frac{1}{j}\right) .
\end{aligned}
$$


Similar estimates are valid for approximated solutions $\rho_{\delta}, u_{\delta}$. More precisely, we have

$$
\left(\rho_{\delta} \log \left(\rho_{\delta}\right)\right)_{t}+\left(\rho_{\delta} \log \left(\rho_{\delta}\right) u_{\delta}\right)_{x}+\rho_{\delta}\left(u_{\delta}\right)_{x}=0,
$$

in $\mathcal{D}^{\prime}((0,1) \times(0, T))$, and

$$
\int_{0}^{T} \int_{0}^{1} \rho_{\delta}\left(u_{\delta}\right)_{x}=\int_{0}^{1} \rho_{0}^{\delta} \log \left(\rho_{0}^{\delta}\right)-\int_{0}^{1} \rho_{\delta} \log \left(\rho_{\delta}\right)(T)
$$

Since $\rho_{\delta} \in L^{\infty}\left(0, T ; L^{\gamma}\right)$, we have

$$
\rho^{\delta} \log \left(\rho^{\delta}\right) \in L^{\infty}\left(0, T ; L^{\tilde{\gamma}}\right)
$$

for $1<\tilde{\gamma}<\gamma$. By the equation (5.44), we obtain

$$
\left(\rho_{\delta} \log \left(\rho_{\delta}\right)\right)_{t} \in L^{\frac{2 \gamma}{\gamma+1}}\left(0, T ; W^{-1, \frac{2 \gamma}{\gamma+1}}\right) .
$$

By Lemma 5.2, we conclude as $\delta \rightarrow 0$

$$
\rho^{\delta} \log \left(\rho^{\delta}\right) \rightarrow \overline{\rho \log (\rho)}, \quad \text { in } C\left([0, T] ; L^{\tilde{\gamma}}-\omega\right) .
$$

This implies

$$
\lim _{\delta \rightarrow 0} \int_{0}^{1} \rho^{\delta} \log \left(\rho^{\delta}\right)(T)=\int_{0}^{1} \overline{\rho \log (\rho)}(T) .
$$

Since the function $x \log (x)$ is convex for any $x>0$, Lemma 5.4 implies that

$$
\rho \log (\rho) \leq \overline{\rho \log (\rho)}, \quad \text { a.e. in }(0,1) \times(0, T) .
$$

Subtracting (5.43) by (5.45) and sending $\delta \rightarrow 0$, we have

$$
\begin{aligned}
& \int_{0}^{1} \overline{\rho \log (\rho)}(T)-\int_{0}^{1}\left(\rho+\frac{1}{j}\right) \log \left(\rho+\frac{1}{j}\right)(T) \\
= & \int_{0}^{1} \rho_{0} \log \left(\rho_{0}\right)-\int_{0}^{1}\left(\rho_{0}+\frac{1}{j}\right) \log \left(\rho_{0}+\frac{1}{j}\right) \\
& +\int_{0}^{T} \int_{0}^{1} \rho(u)_{x}-\lim _{\delta \rightarrow 0} \int_{0}^{T} \int_{0}^{1} \rho_{\delta}\left(u_{\delta}\right)_{x}-\frac{1}{j} \int_{0}^{T} \int_{0}^{1} u_{x} \log \left(\rho+\frac{1}{j}\right) .
\end{aligned}
$$

The first two terms of right hand side can be estimated as follows

$$
\begin{aligned}
& \int_{0}^{T} \int_{0}^{1} \rho(u)_{x}-\lim _{\delta \rightarrow 0} \int_{0}^{T} \int_{0}^{1} \rho_{\delta}\left(u_{\delta}\right)_{x} \\
= & \int_{0}^{T} \int_{0}^{1} \rho(u)_{x}-\lim _{\delta \rightarrow 0} \int_{0}^{T} \int_{0}^{1} \rho_{\delta} \mathcal{H}_{\delta}^{1}-\lim _{\delta \rightarrow 0} \int_{0}^{T} \int_{0}^{1} A_{11}^{-1}\left(n_{\delta}\right) \rho_{\delta}^{\gamma+1} \\
= & \int_{0}^{T} \int_{0}^{1} \rho(u)_{x}-\int_{0}^{T} \int_{0}^{1} \rho \mathcal{H}^{1}-\lim _{\delta \rightarrow 0} \int_{0}^{T} \int_{0}^{1} A_{11}^{-1}\left(n_{\delta}\right) \rho_{\delta}^{\gamma+1} \\
= & \int_{0}^{T} \int_{0}^{1} \rho A_{11}^{-1}(n) \overline{\rho^{\gamma}}-\lim _{\delta \rightarrow 0} \int_{0}^{T} \int_{0}^{1} A_{11}^{-1}(n) \rho_{\delta}^{\gamma+1}-\lim _{\delta \rightarrow 0} \int_{0}^{T} \int_{0}^{1}\left(A_{11}^{-1}\left(n_{\delta}\right)-A_{11}^{-1}(n)\right) \rho_{\delta}^{\gamma+1} \\
= & \lim _{\delta \rightarrow 0} \int_{0}^{T} \int_{0}^{1} A_{11}^{-1}(n)\left(\rho \overline{\rho^{\gamma}}-\rho_{\delta}^{\gamma+1}\right),
\end{aligned}
$$


where we have used Lemma 5.3 in the second equality, and (5.15), $\gamma>1$, and (3.20) in the last step. Here $\mathcal{H}^{1}$ is the first element of $\mathcal{H}$, and $A_{11}^{-1}(\cdot)$ is the $(1,1)$ element of inverse matrix $A^{-1}(\cdot)$. By the estimate (5.25) and the property of $2 \times 2$ matrices, $A_{11}^{-1}(\cdot)>0$.

Since $\rho, \rho_{\delta} \geq 0$, it is not hard to verify that

$$
\left(\rho-\rho_{\delta}\right)^{\gamma+1}=\left(\rho-\rho_{\delta}\right)^{\gamma}\left(\rho-\rho_{\delta}\right) \leq\left(\rho^{\gamma}-\rho_{\delta}^{\gamma}\right)\left(\rho-\rho_{\delta}\right)
$$

Thus

$$
\begin{aligned}
& \varlimsup_{\delta \rightarrow 0} \int_{0}^{T} \int_{0}^{1} A_{11}^{-1}(n)\left(\rho-\rho_{\delta}\right)^{\gamma+1} \\
\leq & \lim _{\delta \rightarrow 0} \int_{0}^{T} \int_{0}^{1} A_{11}^{-1}(n)\left(\rho^{\gamma}-\rho_{\delta}^{\gamma}\right)\left(\rho-\rho_{\delta}\right) \\
= & \lim _{\delta \rightarrow 0} \int_{0}^{T} \int_{0}^{1} A_{11}^{-1}(n)\left(\rho^{\gamma+1}-\rho^{\gamma} \rho_{\delta}-\rho_{\delta}^{\gamma} \rho+\rho_{\delta}^{\gamma+1}\right) \\
= & \lim _{\delta \rightarrow 0} \int_{0}^{T} \int_{0}^{1} A_{11}^{-1}(n)\left(\rho_{\delta}^{\gamma+1}-\rho \overline{\rho^{\gamma}}\right)+\lim _{\delta \rightarrow 0} \int_{0}^{T} \int_{0}^{1} A_{11}^{-1}(n)\left(\rho^{\gamma+1}-\rho^{\gamma} \rho_{\delta}-\rho_{\delta}^{\gamma} \rho+\rho \overline{\rho^{\gamma}}\right) \\
= & \lim _{\delta \rightarrow 0} \int_{0}^{T} \int_{0}^{1} A_{11}^{-1}(n)\left(\rho_{\delta}^{\gamma+1}-\rho \overline{\rho^{\gamma}}\right) .
\end{aligned}
$$

Substituting (5.50) into (5.49), we have

$$
\int_{0}^{T} \int_{0}^{1} \rho(u)_{x}-\lim _{\delta \rightarrow 0} \int_{0}^{T} \int_{0}^{1} \rho_{\delta}\left(u_{\delta}\right)_{x} \leq 0 .
$$

Combing this inequality with (5.48), we conclude that

$$
\begin{aligned}
& \int_{0}^{1} \overline{\rho \log (\rho)}(T)-\int_{0}^{1}\left(\rho+\frac{1}{j}\right) \log \left(\rho+\frac{1}{j}\right)(T) \\
\leq & \int_{0}^{1} \rho_{0} \log \left(\rho_{0}\right)-\int_{0}^{1}\left(\rho_{0}+\frac{1}{j}\right) \log \left(\rho_{0}+\frac{1}{j}\right)-\frac{1}{j} \int_{0}^{T} \int_{0}^{1} u_{x} \log \left(\rho+\frac{1}{j}\right) .
\end{aligned}
$$

Sending $j \rightarrow \infty$, we obtain that

$$
\int_{0}^{1} \overline{\rho \log (\rho)}(T)-\int_{0}^{1} \rho \log (\rho)(T) \leq 0
$$

This and (5.47) imply that $\overline{\rho \log (\rho)}=\rho \log (\rho)$, combined with (5.46), implies (5.40).

Combining Lemma 5.5 with Lemma 5.4, and using the strict convexity of $\rho \log \rho$ for $\rho \geq 0$, we know that

$$
\rho_{\delta} \rightarrow \rho, \quad \text { a.e. in }(0,1) \times(0, T) \text {. }
$$

It follows from the Egorov theorem that for any $\epsilon>0$, there is $I_{\epsilon} \subset(0,1) \times(0, T)$ such that $\left|((0,1) \times(0, T)) \backslash I_{\epsilon}\right|<\epsilon$ and

$$
\sup _{(x, t) \in I_{\epsilon}}\left|\rho_{\delta}(x, t)-\rho(x, t)\right| \rightarrow 0 .
$$


Since $\rho_{\delta}$ is uniformly bounded in $L^{2 \gamma}$, we can estimate

$$
\begin{aligned}
& \int_{0}^{T} \int_{0}^{1}\left|\rho_{\delta}-\rho\right|^{\gamma} \leq \sup _{(x, t) \in I_{\epsilon}}\left|\rho_{\delta}(x, t)-\rho(x, t)\right|\left|I_{\epsilon}\right|+C\left|((0,1) \times(0, T)) \backslash I_{\epsilon}\right|^{\frac{1}{2}}\left\|\rho_{\delta}-\rho\right\|_{L^{2 \gamma}}^{\gamma} \\
& \rightarrow 0, \quad \text { as } \delta \rightarrow 0 .
\end{aligned}
$$

This implies that $\overline{\rho^{\gamma}}=\rho^{\gamma}$ in $(0,1) \times(0, T)$. This completes the proof of Lemma 5.5 .

\section{References}

[1] F. de Anna and C. Liu, Non-isothermal General EricksenLeslie System: Derivation, Analysis and Thermodynamic Consistency. Arch. Ration. Mech. Anal. 231 (2019), 637-717.

[2] S. J. Ding, J. R. Huang, F. G. Xia, H. Y. Wen, R. Z. Zi, Incompressible limit of the compressible nematic liquid crystal flow. J. Funct. Anal. 264 (7) (2013), 1711-1756.

[3] S. J. Ding, J. Y. Lin, C. Y. Wang, and H. Y. Wen, Compressible hydrodynamic flow of liquid crystals in 1D. Discrete and Continuous Dynamical Systems-A 32 (2012), no. 2, 539-563.

[4] S. J. Ding, C. Y. Wang, and Y. H. Wen, Weak solution to compressible hydrodynamic flow of liquid crystals in 1D. Discrete and Continuous Dynamical Systems-B 15 (2011), no. 2, 357-371.

[5] J. L. Ericksen, Hydrostatic theory of liquid crystals. Arch. Ration. Mech. Anal. 9 (1962), 371-378.

[6] E. Feireisl, Dynamics of Viscous Compressible Fluids. Oxford University Press, Oxford, 2004.

[7] E. Feireisl, A. Novotný, and H. Petzeltová, On the existence of globally defined weak solutions to the Navier-Stokes equations. J. Math. Fluid Mech. 3 (2001), 358-392.

[8] F. C. Frank, I. Liquid Crystals. On the theory of liquid crystals. Discussions of the Faraday Society 25 (1958), 19-28.

[9] T. Huang, C. Y. Wang, and H. Y. Wen, Strong solutions of the compressible nematic liquid crystal flow. J. Differential Equations 252 (2012), no. 3, 2222-2265.

[10] T. Huang, C. Y. Wang, and H. Y. Wen, Blow up criterion for compressible nematic liquid crystal flows in dimension three. Arch. Ration. Mech. Anal. 204 (2012), no. 1, 285-311.

[11] F. Jiang, J. Song, and D. H. Wang, On multi-dimensional compressible flows of nematic liquid crystals with large initial energy in a bounded domain. J. Funct. Anal. 265 (2013), no. 12, 3369-3397.

[12] N. Jiang, Y. L. Luo and S. J. Tang, On well-posedness of EricksenLeslies parabolichyperbolic liquid crystal model in compressible flow. Mathematical Models and Methods in Applied Sciences 29 (2019), 01, 121-183. 
[13] S. Jiang, P. Zhang, On spherically symmetric solutions of the compressible isentropic NavierStokes equations. Commun. Math. Phys. 215 (2001), 559-581.

[14] F. M. Leslie, Some thermal effects in cholesteric liquid crystals. Proc. Roy. Soc. A. 307 (1968), 359-372.

[15] F. M. Leslie, Theory of Flow Phenomena in Liquid Crystals. Advances in Liquid Crystals, Vol. 4, 1-81. Academic Press, New York, 1979.

[16] F. H. Lin, Nonlinear theory of defects in nematic liquid crystals: Phase transition and flow phenomena. Comm. Pure Appl. Math. 42 (1989), 789-814.

[17] J. Y. Lin, B. S. Lai, C. Y. Wang, Global finite energy weak solutions to the compressible nematic liquid crystal flow in dimension three, SIAM Jour. Math. Anal. 47 (2015), no. 4, 2952-2983.

[18] P. L. Lions, Mathematical Topics in Fluid Mechanics, Vol. I, Incompressible Models. Clarendon Press, Oxford, 1996.

[19] A. Morro, Modelling of Nematic Liquid Crystals in Electromagnetic Fields. Adv. Theor. Appl. Mech., 2 (2009), no. 1, 43-58.

[20] C. W. Oseen, The theory of liquid crystals. Trans. Faraday Soc. 29 (1933), no. 140, 883-899.

[21] O. Parodi, Stress tensor for a nematic liquid crystal. J. Phys. 31 (1970), 581-584.

[22] J. Simon, Nonhomogeneous viscous incompressible fluids: existence of velocity, density and pressure, SIAM J. Math. Anal. 21(5) (1990), 1093-1117. 